LA-UR 05-8131

\title{
Improved bilinears in lattice QCD with non-degenerate quarks
}

\author{
Tanmoy Bhattacharya and Rajan Gupta \\ Los Alamos National Lab, MS B-285, \\ Los Alamos, New Mexico 87545, USA \\ Weonjong Lee \\ School of Physics, Seoul National University, \\ Seoul, 151-747, Republic of Korea \\ Stephen R. Sharpe and Jackson M. S. Wu* \\ Physics Department, University of Washington, Seattle, WA 98195-1560
}

(Dated: January 7, 2014)

\begin{abstract}
We describe the extension of the improvement program for bilinear operators composed of Wilson fermions to non-degenerate dynamical quarks. We consider two, three and four flavors, and both flavor non-singlet and singlet operators. We find that there are many more improvement coefficients than with degenerate quarks, but that, for three or four flavors, nearly all can be determined by enforcing vector and axial Ward identities. The situation is worse for two flavors, where many more coefficients remain undetermined.
\end{abstract}

\footnotetext{
* Present address: TRIUMF, 4004 Westbrook Mall, Vancouver, BC, V6T 2A3, Canada
} 


\section{INTRODUCTION}

Simulations of lattice QCD with light dynamical quarks are greatly facilitated by the use of improved actions and operators. Calculations are underway using various types of improved fermions - staggered, Domain Wall/overlap, maximally twisted and improved Wilson fermions. Here we focus on improved Wilson fermions, and investigate how the improvement program can be extended to remove errors proportional to $a m_{q}$ ( $a$ is the lattice spacing, and $m_{q}$ a generic quark mass) in the realistic case of non-degenerate quark masses. Of particular interest are the " $N_{f}=2+1$ " theories with $m_{d}=m_{u}<m_{s}$ and " $N_{f}=2+1+1$ " theories with $m_{d}=m_{u}<m_{s}<m_{c}$, and we consider both theories here. As we review below, the improvement of the action for such theories including $a m_{q}$ terms has already been considered, but the improvement of operators has not. As a first step, we consider here the improvement of all quark bilinears. These are of considerable phenomenological interest, since their hadronic matrix elements, combined with experimental results for form factors, determine elements of the CKM matrix. Errors in such hadronic matrix elements proportional to $a m_{s}$ and particularly $a m_{c}$ can be significant, and it is thus important to reduce or remove them.

At present, simulations with staggered fermions are able to reach the smallest dynamical quark masses. This comes at the cost, however, of a multiplication of fermion species, and the concomitant need to use the fourth root of the fermion determinant, so that unitarity can at best be restored in the continuum limit. Wilson fermions have the advantage of a straightforward relation to the continuum theory: each lattice fermion gives rise to a single continuum flavor. They also come, however, with disadvantages: in their original form, the leading discretization errors are of $O(a)$, as compared to $O\left(a^{2}\right)$ for staggered fermions, and these discretization errors explicitly break chiral symmetry. As explained in seminal papers by the ALPHA collaboration [1], one can apply the Symanzik improvement program to Wilson fermions and systematically reduce the errors from $O(a)$ to $O\left(a^{2}\right) .{ }^{1}$ We recall that this requires the addition of all dimension five operators to the action that are consistent with the symmetries of the lattice theory, with their coefficients being determined by appropriate non-perturbative conditions. These conditions are generically chosen to

\footnotetext{
${ }^{1}$ Since the gauge action gives rise to errors of $O\left(a^{2}\right)$ we do not consider its improvement here, although our considerations apply equally well for an improved gauge action, as explained in appropriate places in the following.
} 
enforce a symmetry that is present in the continuum limit but is broken for non-vanishing lattice spacing. For Wilson fermions, the broken symmetries that are used are the flavor non-singlet axial symmetries.

A similar method holds for the improvement of operators. ${ }^{2}$ One must add all operators with the same symmetries having one higher dimension and determine their coefficients by applying appropriate "improvement conditions". This assumes that the operators do not mix with other operators of lower dimension, which will be true in all but one case here.

It is possible to simplify this procedure by considering only the improvement of on-shell quantities - masses, decay constants, physical matrix elements, etc. This allows one to use the equations of motion to reduce the number of higher dimension operators that need be considered. On-shell improvement is equivalent to improving correlation functions in which the arguments are all separated. Off-shell improvement extends this to correlation functions in which some arguments are at the same space-time point, and requires additional contact terms. While we will consider only on-shell improvement here, it will turn out that we need to understand some of the subtleties of off-shell improvement in order to resolve certain puzzles that emerge from our analysis.

The general discussion of Refs. 1] applies in the presence of two or more dynamical quarks. Thus we know from that work how to non-perturbatively improve the action, and it is now standard to implement this in unquenched simulations. Results are available with the Wilson gauge action for two [2, 3] three 3] and four [3] flavors, and also with an improved gauge action [4]. The method of Ref. 1] also allows one to improve the flavor non-singlet axial current in the chiral limit (and a variant of this method has been applied for two flavors in Ref. [5]), and the methodology has been extended to the improvement of non-singlet vector and tensor bilinears in the chiral limit [6, 7, 8]. Theoretical discussion of improvement of non-singlet bilinears away from the chiral limit has been restricted to degenerate quarks [1, 8], or to non-degenerate quarks in the quenched approximation [7, 9].

Here we generalize previous work by considering the improvement of bilinears for the realistic case of non-degenerate quarks. We consider both flavor singlet and non-singlet bilinears; the addition of flavor singlets is required by the analysis, but is also of phenomenological

\footnotetext{
${ }^{2}$ We use the term "improvement" as shorthand for " $O(a)$ improvement" throughout this paper. We do not consider the removal of discretization errors proportional to $a^{2}$ or higher powers.
} 
interest. We explain how working away from the chiral limit introduces a plethora of new improvement constants, and then study which of these can be determined by imposing appropriate axial Ward identities. We find that, for three flavors, nearly all can be determined, with the situation unchanged for four flavors but worse for two flavors. To determine the remaining improvement coefficients one must use other methods, e.g. non-perturbative renormalization [10], suitably improved [1]], or matching short distance correlation functions to perturbative expressions [8].

In this work we need make no specific choice as to how the Ward identities are implemented. One could use a method based on the Schrödinger functional, or use standard hadron correlation functions. Our theoretical discussion holds equally well for either choice. What is key, however, is that one can vary the quark masses independently in a regime where all have $m_{q} a<1$, so that effects proportional to $\left(m_{q} a\right)^{2}$ can be neglected. This condition is satisfied for the physical strange quark for lattice spacings satisfying approximately $a \leq(1 \mathrm{GeV})^{-1}$, and for the physical charm quark if $a \leq(4 \mathrm{GeV})^{-1}$. We stress that these conditions do not necessarily require the quarks to be light (with "light" meaning $\left.m_{q} \ll \Lambda_{\mathrm{QCD}}\right)$.

The practical implementation of our method will be very involved and computationally expensive. An indication of this is that even the simplest step of determining the improvement constant for the non-singlet axial current in the chiral limit has only recently been undertaken for dynamical fermions [5]. For most improvement constants, present calculations instead rely upon one-loop perturbative values. This raises the question of whether our analysis is of purely theoretical interest or will be useful in practice. To ask this another way, will a tree-level or one-loop perturbative estimate of the improvement constants suffice in practice? For example, the mass dependent improvement coefficient for the non-singlet flavor off-diagonal axial current (needed to determine decay constants) enters in an overall factor of $1+a b_{A}\left(m_{j}+m_{k}\right) / 2$. If we use the tree level value of $b_{A}=1$, then the error in this factor is $\sim \alpha_{s} a m_{s} / 2$, assuming a one loop correction to $b_{A}$ of order unity times $\alpha_{s}$. Taking $m_{s} \approx 0.1 \mathrm{GeV}, a^{-1} \approx 2 \mathrm{GeV}$, and $\alpha_{s} \approx 0.3$, the error is less than $1 \%$. This may be smaller than other sources of error, in which case the tree level value for $b_{A}$ would suffice. On the other hand, if we consider $f_{D}$ in the four flavor theory, then the corresponding error is much larger, $\sim 10 \%$, and a more accurate determination of $b_{A}$ is likely needed.

The outline of this paper is as follows. In the next section we recall previous work on 
the improvement of the unquenched theory in the chiral limit. In section III we describe the additional improvement coefficients that are needed in the unquenched theory for nonvanishing quark masses. We then, in section IV] lay out the Ward identities that can be used to determine most of these coefficients. We end by discussing some implications of our results in section $\nabla$. Two appendices present the generalizations to two and four flavors.

This paper is an expansion, clarification and, to some extent, a correction of Ref. [12]. In that work we argued that some of the improvement coefficients could be determined by imposing vector Ward identities. It turns out that this was not correct in all cases, due to the presence of certain contact terms. We explain this point in a final appendix.

A brief summary of the present work has been given in Ref. [13]

\section{REVIEW OF PREVIOUS WORK}

We begin by reviewing previous work on non-perturbative $O(a)$ improvement of unquenched QCD. The ALPHA collaboration [1] has shown how on-shell improvement of the action can be accomplished by adding the Sheikholeslami-Wohlert or "clover" term, with appropriately chosen coefficient $c_{S W}$. Their method requires a flavor non-singlet axial current, and thus works if the number of light quarks, $N_{f}$, is two or greater. Of course, the resulting value of $c_{S W}$ depends on $N_{f}$.

We briefly review the method of Ref. 1], both for completeness and to introduce our notation. One considers matrix elements of the improved axial current and pseudoscalar density, which, in the chiral limit, take the form

$$
\begin{aligned}
& \widehat{A}_{\mu}^{(j k)}=Z_{A} A_{\mu}^{(j k), I} \quad A_{\mu}^{(j k), I}=A_{\mu}^{(j k)}+a c_{A} \partial_{\mu} P^{(j k)} \quad(j \neq k) \\
& \widehat{P}^{(j k)}=Z_{P} P^{(j k), I} \quad P^{(j k), I}=P^{(j k)} \quad(j \neq k) .
\end{aligned}
$$

Here we introduce the notation that we use throughout this article: a "hat" on an operator indicates that it is both $O(a)$ improved and properly normalized, while the superscript "I" indicates improvement alone. The improvement here is on-shell, not off-shell. Flavor indices are shown as superscripts, with $j, k=1-N_{f} . A_{\mu}$ and $P$ are ultra-local lattice transcriptions of the axial current and pseudoscalar density respectively. The simplest choices are exemplified by $A_{\mu}^{(j k)}(x)=\bar{\psi}^{j}(x) \gamma_{\mu} \gamma_{5} \psi^{k}(x)$, with $a^{3 / 2} \psi(x)$ being the bare lattice fermion at site $x$, but our considerations hold for any ultra-local choices. Finally, $\partial_{\mu}$ is an $O(a)$ improved 
lattice derivative, e.g. the symmetric difference divided by $2 a$. Factors of the lattice spacing $a$ are shown explicitly throughout, so that all quantities have the same dimensions as their continuum counterparts.

To determine $c_{S W}$ one enforces the simplest axial Ward identity

$$
\left\langle\partial_{\mu} \widehat{A}_{\mu}^{(j k)}(x)\right\rangle_{J}=\left(\widehat{m}_{j}+\widehat{m}_{k}\right)\left\langle\widehat{P}^{(j k)}(x)\right\rangle_{J}+O\left(a^{2}\right)
$$

Here the $\widehat{m}_{i}$ are improved and normalized quark masses, whose relation to the bare quark masses is discussed below. The subscript on the expectation values indicates that these matrix elements are to be evaluated in the presence of sources, $J$, which are located at different positions from the operators. The sources should have quantum numbers such that the result is non-vanishing, but are otherwise arbitrary (in both form and position). They could be boundary sources in the Schrödinger functional, or standard hadron operators in a traditional large volume calculation. We need not (and do not) specify them. What matters here is that this equation should hold for any such sources (which thus create states with the appropriate quantum numbers in all possible linear combinations). The left- and right-hand sides will only match as the sources are changed (while holding the bare quark masses and couplings fixed so that the $\widehat{m}_{i}$ are fixed) if both $c_{S W}$ and $c_{A}$ are chosen correctly. ${ }^{3}$ Since the accuracy of matching is $O\left(a^{2}\right)$, these constants can only be determined to a relative accuracy of $O(a)$.

An important point concerning the implementation of eq. (3) is that one does not need to know $Z_{A}, Z_{P}$ or the $\widehat{m}_{i}$. One need only require that the ratio of the matrix elements of the improved, but not normalized, quantities, $\left\langle A_{\mu}^{(j k), I}\right\rangle_{J}$ and $\left\langle P^{(j k)}\right\rangle_{J}$, is the same for any choice of $J$. One can then extrapolate the resulting values of $c_{S W}$ and $c_{A}$ to chiral limit. This limit can be determined by setting all $N_{f}$ bare quark masses equal, and extrapolating to the common value for which the right-hand side of eq. (3) vanishes. One then has in hand the desired improvement constants, which will depend, for a given choice of gauge action, only upon the bare coupling constant $g_{0}^{2} \cdot{ }^{4}$

At the same time, one has determined the critical value of hopping parameter, $\kappa_{c}\left(g_{0}^{2}\right)$, as

\footnotetext{
${ }^{3}$ In practice, one can cancel the contributions proportional to one or other of these constants by taking appropriate linear combinations using different sources. This has been done in the determination of $c_{S W}$ in Refs. 2, 3, 4], and of $c_{A}$ in Ref. [5]. This allows one to tune the sources to improve the sensitivity separately for each improvement constant.

${ }^{4}$ In practice, it may be advantageous not to extrapolate $c_{S W}$ and $c_{A}$ to the chiral limit 14]. There would
} 
this is the value for which the right-hand side of eq. (3) vanishes given degenerate quarks. One can then define bare quark masses in the standard way:

$$
a m_{j}=\frac{1}{2 \kappa_{j}}-\frac{1}{2 \kappa_{c}}
$$

These calculations also give information about $Z_{A} / Z_{P}$, but we postpone discussion of this until we have set up the tools to work away from the chiral limit.

The method has been extended, in the chiral limit, to other bilinears. On-shell improvement requires addition of all dimension four operators with appropriate quantum numbers $[1]]^{5}$

$$
\begin{aligned}
& S^{(j k), I}=S^{(j k)} \\
& V_{\mu}^{(j k), I}=V_{\mu}^{(j k)}+a c_{V} \partial_{\nu} T_{\mu \nu}^{(j k)} \\
& T_{\mu \nu}^{(j k), I}=T_{\mu \nu}^{(j k)}+a c_{T}\left[\partial_{\mu} V_{\nu}^{(j k)}-\partial_{\nu} V_{\mu}^{(j k)}\right],
\end{aligned}
$$

where $j \neq k$. Each of these operators also has an associated normalization constant. The improvement constants $c_{V}$ and $c_{T}$ can be determined by enforcing appropriate axial Ward identities in the chiral limit, making use of the previous determination of the improved axial current. In particular, enforcing that the axial variation of $V_{\mu}$ is proportional to $A_{\mu}$ determines $c_{V}$ - see Refs. [6, 7, 8] for particular methods - while enforcing that $T_{\mu \nu}$ rotates into other components of itself determines $c_{T}[7,[]$.

Up to this point we have considered only flavor off-diagonal bilinears, i.e. those with flavor indices satisfying $j \neq k$. For the subsequent analysis we will need to consider also diagonal flavor non-singlet operators (as well as flavor singlets). A convenient common notation for all non-singlet bilinears is $\operatorname{tr}(\lambda \mathcal{O})$, where the trace is over flavor indices, and $\lambda$ is one of the eight Gell-Mann matrices for $N_{f}=3$ (our primary focus), the Pauli matrices for $N_{f}=2$, and the appropriate generalization for $S U(4)$. In the chiral limit, where the $S U\left(N_{f}\right)$ flavor symmetry is unbroken, the improvement of the off-diagonal non-singlets described above carries over also to the diagonal non-singlets, with the same improvement coefficients. Thus, for example,

then be residual $O(a m)$ contributions in these coefficients, but this does not effect $O(a)$ improvement. We find it conceptually simpler, however, to imagine that these coefficients have been extrapolated to the chiral limit, so that they are independent of the quark masses that we will be varying in the subsequent discussion.

${ }^{5}$ Our convention for the tensor is $T_{\mu \nu}^{(j k)}(x)=\bar{\psi}^{j}(x) i \sigma_{\mu \nu} \psi^{k}(x)$ with $\sigma_{\mu \nu}=\frac{1}{2} i\left[\gamma_{\mu}, \gamma_{\nu}\right]$. 
the general improved non-singlet axial current is

$$
\operatorname{tr}\left(\lambda A_{\mu}\right)^{I}=\operatorname{tr}\left(\lambda A_{\mu}\right)+a c_{A} \partial_{\mu} \operatorname{tr}(\lambda P)
$$

for all choices of $\lambda$.

When one moves away from the chiral limit, many new improvement constants are needed. Consider first the gluon action. Possible higher dimension gluonic operators are of dimension six, so the only contribution at $O(a)$ is the original action-density multiplied by the trace of the quark mass matrix, $M$. This leads to an effective gluonic coupling constant [1]

$$
g_{0}^{2} \longrightarrow \widetilde{g}_{0}^{2}=g_{0}^{2}\left(1+a b_{g} \operatorname{tr} M / N_{f}\right)
$$

where $b_{g}$ is a function of $g_{0}^{2}$. If we work at fixed bare coupling, and $\operatorname{vary} \operatorname{tr} M$, then the effective coupling will vary, as will the lattice spacing, and so dimensionful quantities will have $O(a)$ contributions proportional to $a b_{g} \operatorname{tr} M$. To avoid these, $g_{0}$ must, in principle, be varied as $\operatorname{tr} M$ changes in such a way that $\widetilde{g}_{0}$ is held constant. This requires determining the improvement constant $b_{g}$. Non-perturbative methods for doing so have been proposed in Ref. [8], and the one-loop perturbative result is given in Ref. [15].

For most of the following discussion we assume that $b_{g}$ is known, and that whenever we vary $M$ we do so with $\widetilde{g}_{0}$, and thus $a$, fixed. In fact, we will find that our method provides an alternative, independent determination of $b_{g}$. Thus one does not need to rely on the methods of Refs. [1, 8].

The improvement of quark masses and bilinear operators away from the chiral limit in an unquenched theory has been discussed previously, but only in the case of degenerate quarks [1, 8]. We do not recall this work here, since we will generalize it in the following to quarks with non-degenerate masses.

\section{ADDITIONAL IMPROVEMENT COEFFICIENTS}

In this section, we describe the new improvement and renormalization coefficients that are required in order to improve, at $O(a)$, flavor singlet and non-singlet bilinear operators for general values of quark masses. The reason for the inclusion of flavor singlet bilinears will become clear later.

Consider first flavor singlet operators in the chiral limit. Matrix elements of these operators have "quark-disconnected" contractions (in which the operator connects to external 
fields through gluons) in addition to the usual "quark-connected" contractions present also for flavor non-singlet operators. It follows that the improvement coefficients $c_{A}, c_{V}$ and $c_{T}$ will not be the same as those for non-singlet operators, and thus we denote them $\bar{c}_{A}, \bar{c}_{V}$ and $\bar{d}_{T}$, respectively. The differences begin at two-loop, and thus are of $O\left(g_{0}^{4}\right)$ barring unforeseen cancellations. Furthermore, some of these operators can "mix" with purely gluonic operators. Enumerating the available gluonic operators, one finds the following forms for on-shell improved flavor singlet bilinears: ${ }^{6}$

$$
\begin{aligned}
\left(\operatorname{tr} A_{\mu}\right)^{I} & =\operatorname{tr} A_{\mu}+a \bar{c}_{A} \partial_{\mu} \operatorname{tr} P \\
\left(\operatorname{tr} V_{\mu}\right)^{I} & =\operatorname{tr} V_{\mu}+a \bar{c}_{V} \partial_{\nu} \operatorname{tr} T_{\mu \nu} ; \\
\left(\operatorname{tr} T_{\mu}\right)^{I} & =\operatorname{tr} T_{\mu}+a \bar{c}_{T}\left[\partial_{\mu} \operatorname{tr} V_{\nu}-\partial_{\nu} \operatorname{tr} V_{\mu}\right] ; \\
(\operatorname{tr} S)^{I} & =a^{-3} e_{S}+\operatorname{tr} S+a g_{S} \operatorname{Tr}\left(F_{\mu \nu} F_{\mu \nu}\right) ; \\
(\operatorname{tr} P)^{I} & =\operatorname{tr} P+a g_{P} \operatorname{Tr}\left(F_{\mu \nu} \tilde{F}_{\mu \nu}\right) .
\end{aligned}
$$

Here we use "tr" for the trace over flavor indices, and "Tr" for that over color indices. For later convenience, it is important that the discretized form of $\operatorname{Tr}\left(F_{\mu \nu} F_{\mu \nu}\right)$ is exactly that combination of Wilson loops which appears in the gauge action [see eq. (37) below], with an average over loop positions so as to be centered on the site where the bilinear is placed. For $\operatorname{Tr}\left(F_{\mu \nu} \tilde{F}_{\mu \nu}\right)$ one can pick any local choice, e.g. that based on the clover-leaf.

The mixing of $\operatorname{tr} S$ with the identity operator in eq. (13) was overlooked in Ref. [12]. To improve $\operatorname{tr} S$, the coefficient $e_{S}$ would need to be determined to an accuracy of $a^{4}$. To calculate hadronic matrix elements, however, one must subtract disconnected contributions anyway, and this completely removes the $e_{S}$ term. Similarly, the $e_{S}$ contribution can be canceled when implementing Ward identities by subtracting disconnected contributions, as we discuss explicitly below. In this way one can avoid the problem except when calculating the vacuum expectation value, i.e. the quark condensate. To obtain an improved version of the condensate, one must use another method, as also discussed below.

We now turn to improvement away from the chiral limit. We consider explicitly the case of three light dynamical flavors; the generalizations to two and four flavors are described in the

\footnotetext{
${ }^{6}$ In Ref. 12 we erroneously concluded that improvement of the tensor bilinear required the inclusion of an additional gluonic operator, which, however, vanishes identically.
} 
appendices.. We restrict the discussion to a diagonal bare mass matrix with positive entries, $M=\operatorname{diag}\left(m_{1}, m_{2}, m_{3}\right)$, thus avoiding possible phase structure associated with spontaneous violation of CP [16]. Treating $M$ as a spurion transforming in the adjoint representation of the flavor $S U(3)$ group, it is straightforward to enumerate the allowed improvement terms linear in quark masses. For non-singlet operators we find the general form

$$
\widehat{\operatorname{tr}(\lambda \mathcal{O})}=Z_{O}\left[\left(1+a \bar{b}_{O} \operatorname{tr} M\right) \operatorname{tr}(\lambda \mathcal{O})^{I}+a \frac{1}{2} b_{O} \operatorname{tr}(\{\lambda, M\} \mathcal{O})+a f_{O} \operatorname{tr}(\lambda M) \operatorname{tr} \mathcal{O}\right]
$$

where $\mathcal{O}$ is any of the five bilinears. A possible term proportional to $\operatorname{tr}([\lambda, M] \mathcal{O})$ is forbidden by CP invariance - only the anticommutator of $\lambda$ and $M$ appears. Note that the operators appearing in the $O(a)$ corrections on the RHS (right-hand side) can be chosen to be improved or unimproved, the difference being of $O\left(a^{2}\right)$. Here we have left them unimproved for the sake of brevity, but in some equations below it is more convenient use improved versions. This choice has no impact on $Z_{O}$ because the explicit factors of quark mass do not allow mixing back with the leading operator $\mathcal{O}$.

There is one subtlety that is overlooked in eq. (15). The flavor-diagonal scalar bilinears (i.e. those for which the $\lambda$ are diagonal matrices) can also mix with the identity operator. For example, the operator $S^{(j j)}-S^{(k k)}$ mixes with the identity with a coefficient proportional to $\left(m_{j}-m_{k}\right) / a^{2}$. As for the flavor singlet scalars, however, this mixing is removed in all but the vacuum expectation value by subtracting disconnected contributions, and so we will keep its contribution to $S^{(j j), I}-S^{(k k), I}$ implicit in the following.

Aside from this subtlety, there are, for each of the five non-singlet bilinears, three improvement coefficients $b_{O}, \bar{b}_{O}$ and $f_{O}$, in addition to the overall normalization in the chiral limit, $Z_{O}$. It is useful to understand the dependence of each of these quantities on the coupling constant and lattice spacing. In the chiral limit, the normalization depends both on the bare coupling and, if the corresponding bilinear has an anomalous dimension, on $a \mu$ (with $\mu$ the renormalization scale): $Z_{O}=Z_{O}\left(g_{0}^{2}, a \mu\right)$. As discussed in Ref. [1], away from the chiral limit one must replace $g_{0}$ with $\widetilde{g}_{0}$ of eq. (9) so that, in general, $Z_{O}=Z_{O}\left(\widetilde{g}_{0}^{2}, a \mu\right)$. The improvement coefficients do not, however, depend explicitly on $a \mu$, and so are functions only of $g_{0}^{2}$, or equivalently of only $\tilde{g}_{0}^{2}$ at this order of improvement.

To understand the significance of each of the improvement coefficients, it is useful to consider special cases. For flavor off-diagonal operators (which we will also refer to as 
"charged" operators) $f_{O}$ drops out:

$$
\widehat{\mathcal{O}}^{(j k)}=Z_{O}\left[1+a \bar{b}_{O} \operatorname{tr} M+a b_{O} m_{j k}\right] \mathcal{O}^{(j k), I},
$$

where $m_{j k}=\left(m_{j}+m_{k}\right) / 2$. The fact that $\bar{b}_{O}$ multiplies the trace of $M$ (and thus depends on all three quark masses) indicates that it arises from mass dependence of quark loops, and thus begins at two-loop order in perturbation theory, and is absent in the quenched approximation. The $b_{O}$ term, by contrast, arises from the mass dependence of the valence quark propagators attached to the operator, and is present also in the quenched approximation. We have chosen the normalization so as to match that of the standard form used in quenched applications of improvement:

$$
\left.\widehat{\mathcal{O}}^{(j k)}\right|_{\mathrm{Qu}}=Z_{O}\left[1+a b_{O}^{Q} m_{j k}\right] \mathcal{O}^{(j k), I}
$$

Note that, although $b_{O}$ and $b_{O}^{Q}$ arise from the same underlying effect, they will differ numerically (for a given choice of $\tilde{g}_{0}^{2}$ ) due to mass-independent contributions from quark loops. Since these enter first at two loop order, however, one loop results for $b_{O}$ from Ref. 17] are valid also for $b_{O}^{Q}$.

The $f_{O}$ term enters into the improvement of flavor diagonal (or "neutral") operators, e.g.

$$
\begin{aligned}
\widehat{\mathcal{O}}^{(j j)}-\widehat{\mathcal{O}}^{(k k)}=Z_{O}[(1 & \left.+a \bar{b}_{O} \operatorname{tr} M\right)\left(\mathcal{O}^{(j j), I}-\mathcal{O}^{(k k), I}\right)+a b_{O}\left(m_{j} \mathcal{O}^{(j j)}-m_{k} \mathcal{O}^{(k k)}\right) \\
& \left.+a f_{O}\left(m_{j}-m_{k}\right) \operatorname{tr} \mathcal{O}\right]
\end{aligned}
$$

Here, on the left hand side we have made the replacement

$$
\left(\widehat{\mathcal{O}}^{(j j)-\mathcal{O}}(k k)\right)=\widehat{\mathcal{O}}^{(j j)}-\widehat{\mathcal{O}}^{(k k)}
$$

i.e. we have replaced the improved and normalized version of the operator $\left(\mathcal{O}^{(j j)}-\mathcal{O}^{(k k)}\right)$ with the difference of the improved and normalized versions of the individual operators. Similarly, in the first term on the right hand side, we have used

$$
\left(\mathcal{O}^{(j j)}-\mathcal{O}^{(k k)}\right)^{I}=\mathcal{O}^{(j j), I}-\mathcal{O}^{(k k), I}
$$

One might be concerned that there is a subtlety hidden in these replacements, since the individual operators $\mathcal{O}^{(j j)}$ contain flavor singlet parts and, as discussed further below, flavor singlet and non-singlet operators can have different anomalous dimensions. In fact, the same 
issue arises in the continuum. We resolve it by simply defining the individual operators as appropriate sums of the flavor singlet and non-singlet parts, e.g. with two flavors

$$
\widehat{\mathcal{O}}^{(11)} \equiv \frac{1}{2}\left[\left(\mathcal{O}^{(11)-\mathcal{O}^{(22)}}\right)+\left(\mathcal{O}^{(11)+\mathcal{O}^{(22)}}\right)\right]
$$

and similarly for the improved operators. Then the relations in eqs. (19) and (20) are identities.

Returning to eq. (18), it is clear that the $f_{O}$ term arises from quark-disconnected contractions of the operator, because it is only through such contractions that mixing with the operator $\operatorname{tr} \mathcal{O}$, which contains all flavors, can arise. From this we learn that $f_{O}$ appears first at two-loop order. We remark that $f_{O}$ is also present in improvement of quenched bilinears if one considers flavor-diagonal non-singlets and keeps disconnected contractions. As far as we know, no such calculations have been done to date.

The presence of an extra improvement coefficient in the diagonal non-singlet operators as compared to the off-diagonal non-singlets leads to the following apparent paradox. A non-singlet vector rotation $\left(\delta_{V} \psi_{j}=\psi_{k}\right.$ and $\left.\delta_{V} \bar{\psi}_{k}=-\bar{\psi}_{j}\right)$ transforms off-diagonal operators into diagonal ones: $\delta_{V} \mathcal{O}^{(k j)}=\mathcal{O}^{(k k)}-\mathcal{O}^{(j j)}$. Thus the corresponding Ward identity [given explicitly in eq. (C8)] should be enforced for improved operators on the lattice up to $O\left(a^{2}\right)$ corrections. Assuming that $\widehat{\delta_{V} S}$ is known, this identity relates an operator with two improvement coefficients $\left(\bar{b}_{O}\right.$ and $\left.b_{O}\right)$ to an operator with one more improvement coefficient $\left(f_{O}\right)$. Thus it seems to imply that $f_{O}$ is not independent. This would be paradoxical, because $f_{O}$ is allowed in the first place by the vector symmetry which is subsequently leading to the constraint. This paradox is resolved by analyzing the possible contact terms in Appendix C3.

Previous discussions of improvement in the unquenched theory have considered only degenerate quarks [1]. To make contact with the notation used in these papers, we note that the general result (15) reduces to

$$
\left.\widehat{\operatorname{tr}(\lambda \mathcal{O})}\right|_{\mathrm{UnQ}, \text { degen }}=Z_{O}\left[1+a\left(b_{O}+N_{f} \bar{b}_{O}\right) m\right] \operatorname{tr}(\lambda \mathcal{O})^{I}
$$

for degenerate quarks. For charged bilinears, this has the same form as used in Refs. [1], except that what we call $b_{O}+N_{f} \bar{b}_{O}$ was denoted simply $b_{O}$ in those works. We prefer our notation because of its connection with the quenched improvement constants and because 
it is more easily generalizable to non-degenerate quarks. We note that at one-loop order the difference in notation is immaterial because, as noted above, $\bar{b}_{O}$ vanishes at this order.

Next we consider the mass-dependent improvement coefficients needed for flavor singlet operators. We find that the general form consistent with flavor symmetry is

$$
\widehat{\operatorname{tr} \mathcal{O}}=Z_{O} r_{O}\left[\left(1+a \bar{d}_{O} \operatorname{tr} M\right) \operatorname{tr}(\mathcal{O})^{I}+a d_{O} \operatorname{tr}(M \mathcal{O})\right] .
$$

There are only two mass-dependent improvement coefficients for each bilinear (as opposed to the three needed for non-singlets), but there is an additional normalization factor, $r_{O}$, appearing in the chiral limit. The latter arises because the normalizations of singlet and nonsinglet operators differ, since the former can have quark-disconnected contractions. Rather than introduce a new normalization constant $\bar{Z}_{O}$, we have parameterized this effect with the ratio $r_{O}=\bar{Z}_{O} / Z_{O}$. For the axial current $\left(\mathcal{O}=A_{\mu}\right)$ the anomalous dimension of the singlet and non-singlet operators differ (the former beginning at two-loop order, while the latter vanishing to all orders), so that $r_{A}$ must depend explicitly on $\ln a$ in addition to the usual dependence on $\widetilde{g}_{0}^{2}$. For the four other bilinears the singlet and non-singlet anomalous dimensions are the same (the anomalous dimensions vanish for all vector currents, and the quark-disconnected loop diagrams for $S, P$ and $T$ vanish by chirality in the continuum). Thus $r_{S}, r_{P}, r_{V}$ and $r_{T}$ do not depend explicitly on $\ln a$, and are functions only of the effective coupling constants, just like the improvement coefficients. See Ref. [18] for a more thorough discussion of this point.

We also need the expressions for $O(a)$ improved quark masses in terms of the bare quark masses. Using flavor symmetry and the constraint that the singlet and non-singlet mass combinations vanish at the same bare mass [18], we find

$$
\begin{aligned}
& \widehat{\operatorname{tr} \lambda M}=Z_{m}\left[\left(1+a \bar{b}_{m} \operatorname{tr} M\right) \operatorname{tr} \lambda M+a b_{m} \operatorname{tr}\left(\lambda M^{2}\right)\right], \\
& \widehat{\operatorname{tr} M}=Z_{m} r_{m}\left[\left(1+a \bar{d}_{m} \operatorname{tr} M\right) \operatorname{tr} M+a d_{m} \operatorname{tr}\left(M^{2}\right)\right] .
\end{aligned}
$$

The notation is analogous to that used for the bilinears. Note that there is no separate " $f_{O}$-like" term in (24), since such a term, proportional to $\operatorname{tr}(\lambda M) \operatorname{tr}(M)$, can be absorbed into the $\bar{b}_{m}$ term. This reduction in the number of improvement coefficients will play an important role in the next section, where we will see how these constants are related to those needed to improve the scalar bilinear. The overall constant $Z_{m}$ is scale-dependent, but all other constants, including $r_{m}[18]$, are not. 
Since we restrict ourselves to a diagonal mass matrix, the result (24) is non-trivial only for diagonal $\lambda$ 's. Taking appropriate linear combinations with eq. (25), one can obtain the result for individual masses:

$$
\begin{aligned}
\widehat{m}_{j}=\widehat{M}_{j j}=Z_{m}\left\{\left[m_{j}\right.\right. & \left.+\left(r_{m}-1\right) \frac{\operatorname{tr} M}{N_{f}}\right]+a\left[b_{m} m_{j}^{2}+\bar{b}_{m} m_{j} \operatorname{tr} M\right. \\
& \left.\left.+\left(r_{m} d_{m}-b_{m}\right) \frac{\operatorname{tr}\left(M^{2}\right)}{N_{f}}+\left(r_{m} \bar{d}_{m}-\bar{b}_{m}\right) \frac{(\operatorname{tr} M)^{2}}{N_{f}}\right]\right\} .
\end{aligned}
$$

From the $O(1)$ terms we see that $\widehat{m}_{j}$ vanishes if all bare masses vanish together. This is by construction. For non-degenerate positive quark masses, however, $\widehat{m}_{j}$ does not vanish when $m_{j}=0$. This is not an effect which vanishes linearly in $a$, since it remains true even when $O(a)$ terms are dropped, because of the contribution proportional to $\left(r_{m}-1\right) \operatorname{tr} M$. In particular, this effect implies that, at fixed gauge coupling, the pion becomes massless at a value of the bare up and down quark hopping parameters (assumed degenerate) which depends (linearly) on the strange quark mass. This is similar to the well-known result that the partially quenched critical hopping parameter differs from the fully unquenched value (as discussed, e.g., in Ref. [19]).

It is useful to consider which of these constants survive in the quenched approximation. The constants $\bar{b}_{m}$ and $\bar{d}_{m}$ do not, since they are produced by quark loops, as shown by the fact that they multiply $\operatorname{tr}(M)$. Similarly, the difference of $r_{m}$ from unity, and the difference between $d_{m}$ and $b_{m}$, arise from insertions of the mass on quark loops. Thus in the quenched approximation one only needs two constants: $Z_{m}^{Q}$ and $b_{m}^{Q}=d_{m}^{Q}$. One way of seeing this is to note that $\widehat{m}_{j}$ can only depend on $m_{j}$ in the quenched approximation, which forbids all but the first and third terms in eq. (26) .

In summary, moving from degenerate to non-degenerate quarks and considering singlet as well as non-singlet operators requires the introduction of a large number of additional improvement coefficients. All except for $Z_{S} Z_{P}, Z_{T}, Z_{m}$ and $r_{A}$ are scale independent functions of the effective coupling alone, ${ }^{7}$ and for these scale-independent quantities there is no apparent obstacle to their determination using Ward identities. Indeed, as we show in the next section, nearly all can be determined in this way.

\footnotetext{
$7 Z_{S} / Z_{P}$ is scale independent, while $Z_{S} Z_{P}$ is scale dependent.
} 


\section{DETERMINING COEFFICIENTS USING WARD IDENTITIES}

In this section we explain in detail how one can generalize previous methods to determine most of the new improvement and normalization coefficients. We organize our approach into four steps. This is partly as an explanatory aid, but also because some parts of the later steps rely on results from the earlier ones. There remains, however, considerable freedom in the ordering of parts of some steps.

In summary, the four steps are the following. First, we enforce vector charge conservation, which determines most of the improvement coefficients, and the normalization constant, of the vector bilinear. It turns out that this is the only use to which we can put vector Ward identities. In Ref. 12] we had claimed otherwise, but this turns out to be incorrect because of overlooked contact terms. We explain this point in appendix C]

The second step is to relate the improvement and normalization coefficients for the quark masses to those for scalar bilinears. This allows us to use, in step three, the simplest axial Ward identities such as eq. (3) ("two point Ward identities"), to determine combinations

of coefficients for axial, pseudoscalar and scalar bilinears. Finally, in the fourth step we enforce axial Ward identities in which the axial variation occurs in a region including other operators ("three point Ward identities"). Here we have to deal with contact terms.

TABLE I: Relations between renormalization and improvement coefficients for masses and scalar bilinears. LO and NLO indicate leading and next-to-leading order in quark masses. Results are valid for $N_{f}=3$ and 4 . They hold also for $N_{f}=2$ if $b_{S}$ and $b_{m}$ are set to zero.

Order in $M$

LO

NLO
Relationship or constraint

$$
\begin{aligned}
& Z_{S}=1 / Z_{m}, r_{S}=1 / r_{m}, g_{S}=b_{g} /\left(2 g_{0}^{2}\right) \\
& b_{S}=-2 b_{m}, \bar{b}_{S}=-\bar{b}_{m}, N_{f} f_{S}=2\left(b_{m}-d_{m}\right), \\
& d_{S}=b_{S}+N_{f} \bar{b}_{S}, d_{S}+N_{f} \bar{d}_{S}=-2\left(d_{m}+N_{f} \bar{d}_{m}\right)
\end{aligned}
$$


TABLE II: Normalization and improvement coefficients determined using various Ward identities (which are denoted schematically) for $N_{f}=3$. LO and NLO indicate leading and next-to-leading order in quark masses. For completeness, we indicate which Ward identities determine $c_{S W}, c_{V}$, $c_{A}$ and $c_{T}$. For the last nine Ward identities (those below the double line), we assume that the on-shell improved flavor non-singlet axial variation of the action has been determined using the previous identities (i.e. those above the double line). The notation "Not new" in the final two lines indicates that these two identities are equivalent, in the chiral limit, to those considered previously.

\begin{tabular}{|c|c|c|}
\hline Ward identity & $\mathrm{LO}$ & NLO \\
\hline$\left\langle H\left|\sum_{\vec{x}} \widehat{V}_{4}^{(j j)}\right| H\right\rangle=Q_{H}^{j}$ & $Z_{V}, r_{V}$ & $b_{V}, \bar{b}_{V}, f_{V}, d_{V}, \bar{d}_{V}$ \\
\hline$\partial_{\mu} \widehat{A}_{\mu}^{(j k)}=\left(\widehat{m}_{j}+\widehat{m}_{k}\right) \widehat{P}^{(j k)}$ & $Z_{m} Z_{P} / Z_{A}, r_{m}$ & $b_{A}, f_{A}, \quad b_{P}-2 b_{m}, \quad b_{m}+2 r_{m} d_{m}$ \\
\hline and & $r_{P}, g_{P}$ & $\left(1+2 r_{m}\right)^{2} b_{m}+6 r_{m}\left(\bar{b}_{m}-\bar{d}_{m}\right)$ \\
\hline$\partial_{\mu}\left(\widehat{A}_{\mu}^{(j j)}-\widehat{A}_{\mu}^{(k k)}\right)$ & {$\left[c_{S W}, c_{A}\right]$} & $2\left(2+r_{m}\right) b_{m}+3\left(\bar{b}_{P}-\bar{b}_{A}+\bar{b}_{m}\right)$ \\
\hline$=2 \widehat{m}_{j} \widehat{P}^{(j j)}-2 \widehat{m}_{k} \widehat{P}^{(k k)}$ & & $b_{P}+2 r_{P} d_{P}, \quad\left(2+r_{P}\right) b_{P}+6 f_{P}$ \\
\hline & & $r_{P}\left(2+r_{P}\right)\left(\bar{b}_{P}-\bar{d}_{P}\right)-\left(1+2 r_{P}+3 r_{m} r_{P}\right) f_{P}$ \\
\hline$\delta_{A}^{(i j)} T^{(j k)}=T^{(i k)}$ & $Z_{A},\left[c_{T}\right]$ & $b_{T}, 3 \bar{b}_{A}-b_{A}\left(r_{m}-1\right)$ \\
\hline$\delta_{A}^{(i j)} V^{(j k)}=A^{(i k)}$ & $Z_{V}, Z_{A}^{2},\left[c_{V}\right]$ & $b_{A}+b_{V}, 3 \bar{b}_{A}-b_{A}\left(r_{m}-1\right)$ \\
\hline and $V \leftrightarrow A$ & & $6\left(\bar{b}_{A}-\bar{b}_{V}\right)+\left(b_{A}-b_{V}\right)\left(2+r_{m}\right)$ \\
\hline$\delta_{A}^{(i j)} P^{(j k)}=S^{(i k)}$ & $Z_{S} / Z_{P}, Z_{A}^{2}$ & $b_{S}+b_{P}, 3 \bar{b}_{A}-b_{A}\left(r_{m}-1\right)$ \\
\hline and $P \leftrightarrow S$ & & $6\left(\bar{b}_{P}-\bar{b}_{S}\right)+\left(b_{P}-b_{S}\right)\left(2+r_{m}\right)$ \\
\hline$\delta_{A}^{(i j)} \operatorname{tr} A=\delta_{A}^{(i j)} \operatorname{tr} V=0$ & $\bar{c}_{A}, \bar{c}_{V}$ & $d_{A}, d_{V}$ \\
\hline$\delta_{A}^{(i j)} \operatorname{tr} P=2 S^{(i j)}$ & $Z_{P} r_{P} / Z_{S}, g_{P}$ & $d_{P}, 3\left(\bar{b}_{S}-\bar{d}_{P}\right)-b_{S}\left(r_{m}-1\right)$ \\
\hline$\delta_{A}^{(i j)} \operatorname{tr} T=2 T^{(i j)}$ & $r_{T}, \bar{c}_{T}$ & $d_{T}, 3\left(\bar{b}_{T}-\bar{d}_{T}\right)-b_{T}\left(r_{m}-1\right)$ \\
\hline$\delta_{A}^{(i j)} \operatorname{tr} S=2 P^{(i j)}$ & $Z_{S} r_{S} / Z_{P}, g_{S}$ & $d_{S}, 3\left(\bar{b}_{P}-\bar{d}_{S}\right)-b_{P}\left(r_{m}-1\right)$ \\
\hline$\delta_{A}^{(i j)} T^{(j i)}=T^{(i i)}+T^{(j j)}$ & $r_{T}, \bar{c}_{T},\left[c_{T}\right]$ & $b_{T}, d_{T}, r_{T}\left(\bar{d}_{T}-\bar{b}_{T}\right)-r_{m} f_{T}$ \\
\hline$\delta_{A}^{(i j)} S^{(j i)}=P^{(i i)}+P^{(j j)}$ & $Z_{P} / Z_{S}, r_{P}, r_{S}$ & $b_{P}+b_{S}, 2 r_{P} d_{P}+b_{P}, 2 r_{S} d_{S}+b_{S}$ \\
\hline and $S \leftrightarrow P$ & $g_{P}, g_{S}$ & $6\left(\bar{b}_{P}-\bar{b}_{S}\right)+\left(2+r_{m}\right)\left(b_{P}-b_{S}\right)$ \\
\hline & & $3 r_{P}\left(\bar{d}_{P}-\bar{b}_{S}\right)+r_{P}\left(d_{P}-b_{S}\right)-r_{m}\left(3 f_{P}+b_{P}-r_{P} b_{S}\right)$ \\
\hline & & $3 r_{S}\left(\bar{d}_{S}-\bar{b}_{P}\right)+r_{S}\left(d_{S}-b_{P}\right)-r_{m}\left(3 f_{S}+b_{S}-r_{S} b_{P}\right)$ \\
\hline$\delta_{A}^{(i j)} A^{(j i)}=V^{(j j)}-V^{(i i)}$ & Not new & $\bar{b}_{V}, b_{V}, f_{V}$ \\
\hline$\delta_{A}^{(i j)} V^{(j i)}=A^{(j j)}-A^{(i i)}$ & Not new & $\bar{b}_{A}, b_{A}, f_{A}$ \\
\hline
\end{tabular}




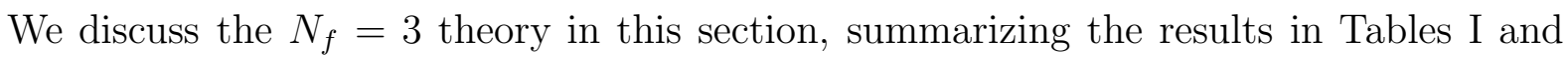
II. The former collects the relationships between constants, while the latter shows which can be obtained from which Ward identities. The generalizations to $N_{f}=4$ and $N_{f}=2$ are discussed, respectively, in appendices $\mathrm{A}$ and $\mathrm{B}$

\section{A. Vector Ward identities}

We use the standard method of enforcing the correct normalization of the vector charge,

$$
\left\langle H\left|\widehat{\mathcal{Q}}^{j}(\tau)\right| H\right\rangle=Q_{H}^{j}\langle H \mid H\rangle, \quad \widehat{\mathcal{Q}}^{j}(\tau)=\sum_{\vec{x}} \widehat{V}_{4}^{(j j)}(\vec{x}, \tau)
$$

where $Q_{H}^{j}$ is the j'th quark number of hadron $\mathrm{H}$, for a convenient set of hadrons. In fact, one does not have to project onto a single hadron - any linear combination created by an operator with a given j'th quark number will work. As we now show, this method can determine all improvement and renormalization coefficients for the vector current, with the exception of $c_{V}$ and $\bar{c}_{V}$. These two are excluded because the operators they multiply vanish at zero spatial momentum.

To see that all other coefficients can be determined we use eqs. (18) and (23) with $\mathcal{O}=V_{4}$ at zero spatial momentum. Consider first degenerate quarks, so that

$$
\begin{aligned}
\widehat{\mathcal{Q}}^{j}-\widehat{\mathcal{Q}}^{k} & =Z_{V}\left[1+a\left(3 \bar{b}_{V}+b_{V}\right) m\right]\left(\mathcal{Q}^{j}-\mathcal{Q}^{k}\right) \\
\widehat{\operatorname{tr} \mathcal{Q}} & =Z_{V} r_{V}\left[1+a\left(3 \bar{d}_{V}+d_{V}\right) m\right] \operatorname{tr} \mathcal{Q}
\end{aligned}
$$

where $\mathcal{Q}^{j}=\sum_{\vec{x}} V_{4}^{(j j)}$ is the bare charge operator. We have dropped the superscript $I$ since the $c_{V}$ and $\bar{c}_{V}$ terms do not contribute. Enforcing the normalization of these two charges for two or more values of the common quark mass determines $Z_{V}, 3 \bar{b}_{V}+b_{V}, r_{V}$ and $3 \bar{d}_{V}+d_{V}$. Note that for the singlet charge operator one must use baryonic states, so that the total charge is non-zero. To obtain the remaining mass-dependent improvement coefficients non-degenerate quarks are required. It is sufficient, however, to work with a " $2+1$ " flavor theory, i.e. one in which the up and down quarks are degenerate (with mass $m_{1}$ ) but have a different mass than the strange quark $\left(m_{3}\right)$. In this case, the improvement term for the flavor non-singlet charge $\widehat{\mathcal{Q}}^{1}-\widehat{\mathcal{Q}}^{3}$ is proportional to

$$
a\left\{\left[\left(2 \bar{b}_{V}+b_{V}\right) m_{1}+\bar{b}_{V} m_{3}\right] \mathcal{Q}^{1}-\left[2 \bar{b}_{V} m_{1}+\left(\bar{b}_{V}+b_{V}\right) m_{3}\right] \mathcal{Q}^{3}+f_{V}\left(m_{1}-m_{3}\right) \operatorname{tr} \mathcal{Q}\right\}
$$


as can be seen from (18). Thus by varying $m_{1}$ at fixed $m_{3}$, and considering different hadrons so that the contributions of the independent operators $\mathcal{Q}^{1}, \mathcal{Q}^{3}$ and $\operatorname{tr} \mathcal{Q}$ vary, one can determine $b_{V}, \bar{b}_{V}$ and $f_{V}$ separately. ${ }^{8}$ Similarly, the improvement term for the singlet charge is proportional to

$$
a\left\{\left(2 \bar{d}_{V}+d_{V}\right) m_{1}\left(\mathcal{Q}^{1}+\mathcal{Q}^{2}\right)+\left(\bar{d}_{V}+d_{V}\right) m_{3} \mathcal{Q}^{3}\right\}
$$

so that $d_{V}$ and $\bar{d}_{V}$ can be disentangled by varying $m_{1}$.

In practice, $Z_{V}$ and $3 \bar{b}_{V}+b_{V}$ have been computed for two flavors using this method [20] (although recall that the latter combination is referred to as $b_{V}$ in Ref. [20]). Determination of $b_{V}$ and $\bar{b}_{V}$ separately should be relatively straightforward, since quark-disconnected contractions are not required. By contrast, the determination of $r_{V}, \bar{d}_{V}, d_{V}$ and $f_{V}$ requires such contractions, and will thus be more challenging in practice. ${ }^{9}$

Vector Ward identities cannot be used to determine any of the other normalization or improvement coefficients, essentially because the vector symmetries are not broken by the discretization. We discuss this further in Appendix C, because in some cases it is not immediately obvious why vector Ward identities cannot be used. Indeed, in Ref. [12], we argued that it was possible to use such identities, and it is instructive to see the flaw in our argument.

\section{B. Relating improvement of mass and scalar bilinear}

As is well known, the anomalous dimensions of the quark mass and scalar bilinear are equal in magnitude but opposite in sign, and it is conventional and convenient to choose their renormalization constants to be the inverse of one another, $Z_{S} Z_{m}=1$. While not strictly necessary (the right hand side could be a constant other than unity), this choice implies that derivatives with respect to quark mass give rise to insertions of the (space-time integral) of the scalar bilinear both for bare and renormalized quantities. In particular, it

\footnotetext{
${ }^{8}$ Determination of $b_{V}$ and $\bar{b}_{V}$ alone can be done using $\mathcal{Q}^{1}-\mathcal{Q}^{2}$, for which the $f_{V}$ term vanishes in the $2+1$ theory.

${ }^{9}$ One can show that the quark-disconnected diagrams give vanishing contributions if one uses the charge built out of the conserved vector current on the lattice. We can see no argument to extend this result to choices of the bare current which are not exactly conserved.
} 
implies that the useful result

$$
\left.\frac{\partial m_{H}}{\partial m_{j}}\right|_{m_{k \neq j}}=\frac{V\left\langle H\left|S^{(j j)}\right| H\right\rangle}{\langle H \mid H\rangle}
$$

(where $|H\rangle$ is the state containing an arbitrary hadron of mass $m_{H}$ at rest, $V$ is the spatial volume, and $k$ runs over the flavors different from $j$ ) holds for both bare and renormalized quantities.

In this section we apply the condition (32) as a constraint not only in the continuum limit but also for non-vanishing lattice spacing. The argument for doing so is straightforward: the quantities appearing in the relation- $\left\langle H\left|S^{(j j)}\right| H\right\rangle, m_{H}$ and $m_{j}$ - are physical quantities that should be improved with appropriate choices of the improvement coefficients introduced above. Thus if the relation (32) holds in the continuum limit it should receive no $O(a)$ corrections once improvement has been implemented. Perhaps surprisingly, this simple relation leads to a number of non-trivial constraints.

The precise relation we enforce is ${ }^{10}$

$$
\left.\frac{\partial m_{H}}{\partial \widehat{m}_{j}}\right|_{\widehat{m}_{k \neq j}, a}=\frac{V\left\langle H\left|\widehat{S}^{(j j)}\right| H\right\rangle}{\langle H \mid H\rangle} .
$$

Here we have been specific about the meaning of the partial derivative in the presence of the regulator. It should be taken with the regulator-here the lattice spacing $a$ - fixed, so that the relation survives in the limit that the regulator is removed. In order to match the formal result (32) the derivative should also be taken with the other improved (rather than bare) masses held fixed. Finally, note that the matrix element of $\widehat{S}$ is, as usual, the connected matrix element, so that the part of $\widehat{S}$ proportional to the identity [the $e_{S}$ term in eq. (13)] does not contribute.

Despite the fact that it involves the a priori unknown quantities $\left\langle H\left|\widehat{S}^{(j j)}\right| H\right\rangle$, this relation is useful because we do know how hadron masses depend on bare parameters. In particular, because the fermion action depends on the bare quark masses only as

$$
S_{l a t, F}=\sum_{x} \sum_{j}\left(a m_{j}\right)\left(a^{3} S^{(j j)}[x]\right)+\ldots
$$

${ }^{10}$ We stress that all the lattice quantities appearing in this relation are defined to have the same dimension as their continuum counterparts, e.g. $a m_{H}$ is the hadron mass in lattice units. 
(even after improvement by the addition of the clover term) one can show that [21]

$$
\left.\frac{\partial\left(a m_{H}\right)}{\partial\left(a m_{j}\right)}\right|_{(a m)_{k \neq j}, g_{0}^{2}}=\frac{L^{3}\left\langle H\left|\left(a^{3} S^{(j j)}\right)\right| H\right\rangle}{\langle H \mid H\rangle}=\frac{V\left\langle H\left|S^{(j j)}\right| H\right\rangle}{\langle H \mid H\rangle} .
$$

In the derivative on the LHS (left-hand side) the bare coupling and the other bare quark masses in lattice units are held fixed. As mentioned above, it is the connected matrix element of the scalar density which appears on the RHS. Similarly, one finds

$$
-\left.2 g_{0}^{4} \frac{\partial\left(a m_{H}\right)}{\partial g_{0}^{2}}\right|_{a m_{j}}=\frac{a V\left\langle H\left|\operatorname{Tr}\left(F_{\mu \nu} F_{\mu \nu}\right)\right| H\right\rangle}{\langle H \mid H\rangle} .
$$

where we define the discretized field strength from the specific form of the gluon action being used:

$$
S_{l a t, G} \equiv a^{4} \sum_{x} \frac{1}{2 g_{0}^{2}} \operatorname{Tr}\left(F_{\mu \nu} F_{\mu \nu}\right)(x) .
$$

In this way, our expressions hold for any choice of gluon action.

To relate the desired derivative in eq. (333) to those which we know, (35) and (36), we proceed in two stages. First, we relate derivatives with respect to improved masses to those with respect to bare masses, using the properties of Jacobians:

$$
\begin{aligned}
\left.\frac{\partial m_{H}}{\partial \widehat{m}_{j}}\right|_{\widehat{m}_{k}, \widehat{m}_{l}, a} & =\frac{\partial\left(m_{H}, \widehat{m}_{k}, \widehat{m}_{l}\right)}{\partial\left(\widehat{m}_{j}, \widehat{m}_{k}, \widehat{m}_{l}\right)} \\
& =\frac{\partial\left(m_{H}, \widehat{m}_{k}, \widehat{m}_{l}\right) / \partial\left(m_{j}, m_{k}, m_{l}\right)}{\partial\left(\widehat{m}_{j}, \widehat{m}_{k}, \widehat{m}_{l}\right) / \partial\left(m_{j}, m_{k}, m_{l}\right)},
\end{aligned}
$$

where $k$ and $l$ are the two flavor indices not equal to $j$. Here all derivatives are at fixed $a$, or equivalently at fixed $\tilde{g}_{0}^{2}$. This means that the derivatives such as $\partial \widehat{m}_{k} / \partial m_{j}$ can be straightforwardly evaluated using eq. (26) . The renormalization constants $Z_{m}$ and $r_{m}$ are functions of $\tilde{g}_{0}^{2}$ and $a$, and thus are fixed. The same is true, to the order in $a$ that we are working, of the improvement constants $b_{m}$ etc., since they can equally well be considered functions of $g_{0}^{2}$ or $\tilde{g}_{0}^{2}$.

Derivatives such as $\partial m_{H} / \partial m_{j}$ cannot yet be evaluated since they are taken at fixed $a$. Using eq. (9), one can relate them to the derivatives we know from eqs. (35) and (36):

$$
\left.\frac{\partial m_{H}}{\partial m_{j}}\right|_{m_{k \neq j}, a}=\left.\frac{\partial\left(a m_{H}\right)}{\partial\left(a m_{j}\right)}\right|_{(a m)_{k \neq j}, a}=\left.\frac{\partial\left(a m_{H}\right)}{\partial\left(a m_{j}\right)}\right|_{(a m)_{k \neq j}, g_{0}^{2}}-\left.\frac{g_{0}^{2} b_{g}}{N_{f}} \frac{\partial\left(a m_{H}\right)}{\partial g_{0}^{2}}\right|_{a m_{j}} .
$$

Putting things together we obtain an expression for the right-hand side of eq. (33) in terms of matrix elements of the bare scalar density and the gluon field strength, and the improvement and normalization constants for quark masses. The left-hand side of eq. (33) 
can be expanded, using eqs. (18) and (23), in terms of the same matrix elements, but with the coefficients being the improvement and normalization constants for the scalar bilinear. Matching the coefficients on the two sides gives, after tedious algebra, the relations quoted in Table 1

We do not go through the details of this calculation in the general case, but do display the subset of the argument that uses only degenerate quarks. In this case, eqs. (38) and (39) simplify to

$$
\begin{aligned}
\left.\frac{\partial m_{H}}{\partial \widehat{m}}\right|_{a} & =\frac{\partial\left(a m_{H}\right) /\left.\partial(a m)\right|_{a}}{\partial \widehat{m} /\left.\partial m\right|_{a}} \\
& =\frac{\partial\left(a m_{H}\right) /\left.\partial(a m)\right|_{g_{0}^{2}}-g_{0}^{2} b_{g} \partial\left(a m_{H}\right) /\left.\partial\left(g_{0}^{2}\right)\right|_{a m}}{\partial \widehat{m} /\left.\partial m\right|_{a}} \\
& =\frac{V\left\langle H\left|\operatorname{tr} S+a\left(b_{g} / 2 g_{0}^{2}\right) \operatorname{Tr}\left(F_{\mu \nu} F_{\mu \nu}\right)\right| H\right\rangle}{Z_{m} r_{m}\left[1+2 a\left(3 \bar{d}_{m}+d_{m}\right) m\right]},
\end{aligned}
$$

where in the last step we have assumed $\langle H \mid H\rangle=1$. This should be equated with the improved matrix element

$$
V\langle H|\operatorname{tr} \widehat{S}| H\rangle=V\left\langle H\left|Z_{S} r_{S}\left\{\left[1+a\left(3 \bar{d}_{S}+d_{S}\right) m\right] \operatorname{tr} S+a g_{S} \operatorname{Tr}\left(F_{\mu \nu} F_{\mu \nu}\right)\right\}\right| H\right\rangle,
$$

where, as noted above, the part of $\widehat{\operatorname{tr} S}$ proportional to the identity operator does not contribute. We conclude that $Z_{S} r_{S}=1 /\left(Z_{m} r_{m}\right), 3 \bar{d}_{S}+d_{S}=-2\left(3 \bar{d}_{m}+d_{m}\right)$ and $b_{g}=2 g_{0}^{2} g_{S}$, which are a subset of the results in Table

We find that the quenched relations $Z_{m} Z_{S}=1$ and $b_{S}=-2 b_{m}$ (Ref. 17]) continue to hold (although the constants themselves will differ from their quenched values), and that there are generalizations for some of the new constants that appear $\left(\bar{b}_{S}=-\bar{b}_{m}\right.$, etc.). There are two particularly interesting, and perhaps unexpected, results. First, there is a constraint on the scalar improvement coefficients $\left(d_{S}=b_{S}+3 \bar{b}_{S}\right)$. This arises because, as noted above, there is one less improvement constant needed for quark masses than for the scalar bilinear (i.e. there is no independent $f_{m}$ term). Second, the relation $b_{g}=2 g_{0}^{2} g_{S}$ provides another way of determining $b_{g}$, if we determine $g_{S}$ using Ward identities as discussed below.

Given the relations of Table I it is interesting to determine if there are any products of masses times scalar densities which maintain their form under improvement. This does not hold for the contribution of mass terms to the action itself, i.e.

$$
\sum_{j} \widehat{m}_{j} \widehat{S}^{(j j)} \neq \sum_{j} m_{j} S^{(j j)} .
$$


The corrections to this equation in the general case of non-degenerate quarks are lengthy and uninformative, so we quote the result only for the case of degenerate quarks

$$
\left.\widehat{m} \operatorname{tr} \widehat{S}\right|_{m_{i}=m_{j}=m_{k}}=m \operatorname{tr} S+a\left(3 \bar{d}_{m}+d_{m}\right) m^{2} \operatorname{tr} S+a g_{S} m \operatorname{Tr}\left(F_{\mu \nu} F_{\mu \nu}\right)
$$

Here we have dropped the term containing the identity operator since it does not contribute to matrix elements. This degenerate quark result illustrates the general point that there is, at $O(a)$, mixing with other terms in the action, and thus no reason to expect that each term in the action should be separately form invariant under improvement. The one example of form invariance is for the variation of the action under vector transformations:

$$
\left(\widehat{m}_{j}-\widehat{m}_{k}\right) \widehat{S}^{(j k)}=\left(m_{j}-m_{k}\right) S^{(j k)}+O\left(a^{2}\right)
$$

This follows from the definitions (15) and (24) and the relations $Z_{S} Z_{m}=r_{S} r_{m}=1, b_{S}=$ $-2 b_{m}$ and $\bar{b}_{S}=-\bar{b}_{m}$. It holds for $N_{f}=2-4$ (and we suspect for all higher $N_{f}$ as well). This relation plays an important role in the discussion of vector Ward identities in Appendix C.

\section{Two-point axial Ward identities}

In this section we investigate which of the improvement and normalization coefficients can be determined using two-point Ward identities such as eq. (3). To obtain as much information as possible we need to vary the quark masses independently (as done in the quenched theory in Ref. [9]) and consider the PCAC relation for both charged and neutral currents.

We assume that $c_{A}$ has been determined, so that we know $A^{(j k), I}$. We can then calculate the Ward identity mass,

$$
\widetilde{m}_{j k} \equiv \frac{\left\langle\partial_{\mu} A_{\mu}^{(j k), I}(x)\right\rangle_{J}}{2\left\langle P^{(j k)}(x)\right\rangle_{J}} \quad(j \neq k) .
$$

Because we have improved the action and the axial current, $\widetilde{m}_{j k}$ should be independent of $x$ and of the source $J$ up to corrections of $O\left(a^{2}\right)$, and thus we do not give it any arguments, nor specify the source. We imagine choosing a source with a good signal, varying the quark masses (keeping $\tilde{g}_{0}^{2}$ fixed, as always) and studying the bare quark mass dependence of $\widetilde{m}_{j k}$. 
Using the Ward identity (3), we have

$$
\begin{aligned}
\widetilde{m}_{j k} & =\frac{1}{2}\left(\widehat{m}_{j}+\widehat{m}_{k}\right) \frac{Z_{P}\left(1+a \bar{b}_{P} \operatorname{tr} M+a b_{P} m_{j k}\right)}{Z_{A}\left(1+a \bar{b}_{A} \operatorname{tr} M+a b_{A} m_{j k}\right)} \\
& =\frac{Z_{P} Z_{m}}{Z_{A}}\left[m_{j k}+\frac{\operatorname{tr} M}{3}\left(r_{m}-1\right)+a\left(\mathcal{A} m_{j k}^{2}+\mathcal{B} m_{j k} \operatorname{tr} M+\mathcal{C} \operatorname{tr}(M)^{2}+\mathcal{D} \operatorname{tr}\left(M^{2}\right)\right)\right] \\
\mathcal{A} & =b_{P}-b_{A}-2 b_{m} \\
\mathcal{B} & =\bar{b}_{P}-\bar{b}_{A}+\bar{b}_{m}+2 b_{m}+\frac{r_{m}-1}{3}\left(b_{P}-b_{A}\right) \\
\mathcal{C} & =\frac{r_{m}-1}{3}\left(\bar{b}_{P}-\bar{b}_{A}\right)+\frac{r_{m} \bar{d}_{m}-\bar{b}_{m}}{3}-\frac{b_{m}}{2} \\
\mathcal{D} & =\frac{2 r_{m} d_{m}+b_{m}}{6} .
\end{aligned}
$$

From the terms linear in bare quark masses one can extract $Z_{m} Z_{P} / Z_{A}$ (as in the quenched case) and $r_{m}$ (absent in the quenched case). For example, the derivative of $\widetilde{m}_{j k}$ with respect to $m$ at $M=0$ for degenerate quarks is $r_{m} Z_{m} Z_{P} / Z_{A}$, while the derivative with respect to $m_{l}, l \neq j, k$ alone is $\left(r_{m}-1\right) Z_{m} Z_{P} /\left(3 Z_{A}\right)$ (a quantity which vanishes in the quenched theory). Using the relations in Table \one has thus determined $Z_{P} /\left(Z_{A} Z_{S}\right)$ and $r_{S}=1 / r_{m}$.

From the quadratic terms one can determine the coefficient of each of the four linearly independent functions of masses that appear. Thus we obtain four linear combinations of the eight constants $b_{A}, \bar{b}_{A}, b_{P}, \bar{b}_{P}, b_{m}, \bar{b}_{m}, d_{m}$ and $\bar{d}_{m}$ (given that we know $r_{m}$ from above). By comparison, in the quenched approximation, where there are only three constants, $b_{A}^{Q}$, $b_{P}^{Q}$ and $b_{m}^{Q}=d_{m}^{Q}$, one can determine the two combinations $b_{A}^{Q}-b_{P}^{Q}$ and $b_{m}^{Q}[9]$.

To obtain further information, we generalize the method by considering flavor-diagonal two-point Ward identities, e.g.

$$
\left\langle\partial_{\mu}\left(\widehat{A}_{\mu}^{(11)}(x)-\widehat{A}_{\mu}^{(22)}\right)(x)\right\rangle_{J}=\left\langle 2 \widehat{m}_{1} \widehat{P}^{(11)}(x)-2 \widehat{m}_{2} \widehat{P}^{(22)}(x)\right\rangle_{J}+O\left(a^{2}\right) .
$$

This introduces several of the new constants present in the unquenched theory: $f_{A}$ on the left-hand side, and $f_{P}, d_{P}$ and $\bar{d}_{P}$ on the right-hand side (which contains the flavor-singlet $\widehat{P}$ ). This corresponds to the fact that, for $m_{1} \neq m_{2}$, there are contractions in which the source $J$ and the axial current (or pseudoscalar density) are not connected by quark propagators. In fact, it is only for $m_{1} \neq m_{2}$ that this identity gives new information: for degenerate quarks, and with appropriate sources, the contractions are exactly the same as those for the flavor off-diagonal identity (3).

To enforce the Ward identity, we have to adjust the constants so that the LHS and RHS are equal for all choices of $x$ and sources $J$, up to $O\left(a^{2}\right)$. We cannot simply take their ratio, 
as we did for the off-diagonal Ward identity, since more than one operator contributes on both sides of the equation, with relative strengths that we do not know a priori. To proceed, it is useful to expand out the operator appearing in the left-hand side of (54):

$$
\begin{aligned}
\text { LHS } & =Z_{A}\left[1+\frac{\left(b_{A}+3 \bar{b}_{A}\right)}{3} \operatorname{tr} M+\frac{b_{A}}{6} \operatorname{tr}\left(\lambda_{8}^{\prime} M\right)\right]\left\{\partial_{\mu} \operatorname{tr}\left(\lambda_{3} A_{\mu}\right)^{I}+a \mathcal{E} \operatorname{tr}\left(\lambda_{3} M\right)\right\} \\
6 \mathcal{E} & =2\left(b_{A}+3 f_{A}\right) \partial_{\mu} \operatorname{tr} A_{\mu}+b_{A} \partial_{\mu} \operatorname{tr}\left(\lambda_{8}^{\prime} A_{\mu}\right) .
\end{aligned}
$$

For convenience we have defined $\lambda_{8}^{\prime}=\sqrt{3} \lambda_{8}$. We can now divide the Ward identity (544) by the overall function of the masses multiplying $\partial_{\mu} \operatorname{tr}\left(\lambda_{3} A_{\mu}\right)^{I}$, so that the operator on the left-hand side becomes

$$
\mathrm{LHS}^{\prime} \equiv \frac{\mathrm{LHS}}{Z_{A}\left[1+\frac{\left(b_{A}+3 \bar{b}_{A}\right)}{3} \operatorname{tr} M+\frac{b_{A}}{6} \operatorname{tr}\left(\lambda_{8}^{\prime} M\right)\right]}=\partial_{\mu} \operatorname{tr}\left(\lambda_{3} A_{\mu}\right)^{I}+a \mathcal{E} \operatorname{tr}\left(\lambda_{3} M\right) .
$$

Since we have previously determined the improvement coefficients in the leading order operator in this equation, $\partial_{\mu} \operatorname{tr}\left(\lambda_{3} A_{\mu}\right)^{I}$, the coefficients of all other, independent, operators appearing on both sides of the rescaled Ward identity can be determined. In particular, we immediately see that $b_{A}+3 f_{A}$ and $b_{A}$ can be determined since they multiply independent operators in $\mathcal{E}$.

We now divide the right-hand side by the same factor, and split the operator which results into terms linear and quadratic in quark masses:

$$
\mathrm{RHS}^{\prime} \equiv \frac{\mathrm{RHS}}{Z_{A}\left[1+\frac{\left(b_{A}+3 \bar{b}_{A}\right)}{3} \operatorname{tr} M+\frac{b_{A}}{6} \operatorname{tr}\left(\lambda_{8}^{\prime} M\right)\right]}=\mathrm{RHS}_{I}^{\prime}+\mathrm{RHS}_{I I}^{\prime}
$$

We find

$$
\begin{aligned}
\operatorname{RHS}_{I}^{\prime}=\frac{Z_{m} Z_{P}}{3 Z_{A}}\{ & \operatorname{tr}\left(\lambda_{3} P\right)\left[2 r_{m} \operatorname{tr} M+\operatorname{tr}\left(\lambda_{8}^{\prime} M\right)\right] \\
& \left.+\operatorname{tr}\left(\lambda_{3} M\right)\left[2 r_{P} \operatorname{tr} P+\operatorname{tr}\left(\lambda_{8}^{\prime} P\right)+2 a r_{P} g_{P} \operatorname{Tr}\left(F_{\mu \nu} \widetilde{F}_{\mu \nu}\right)\right]\right\} .
\end{aligned}
$$

We can determine the coefficients of each independent function of masses (of which there are three at this order) multiplying each independent operator. Thus we can determine $Z_{m} Z_{P} / Z_{A}$ and $r_{m}$ again, as well as $r_{P}$ and $g_{P}$ for the first time.

The quadratic terms are more complicated. There are six independent functions of the masses, each potentially multiplying three independent operators, although the $1 \leftrightarrow 2$ anti- 
symmetry allows only eight independent products. We find

$$
\begin{aligned}
\mathrm{RHS}_{I I}^{\prime}=a \frac{Z_{m} Z_{P}}{9 Z_{A}}\left\{\left[\mathcal{F} \operatorname{tr}(M)^{2}+\mathcal{G} \operatorname{tr}\left(\lambda_{3} M\right)^{2}+\mathcal{H} \operatorname{tr}(M) \operatorname{tr}\left(\lambda_{8}^{\prime} M\right)+\mathcal{I} \operatorname{tr}\left(M^{2}\right)\right] \operatorname{tr}\left(\lambda_{3} P\right)\right. \\
+\left[\mathcal{J} \operatorname{tr}\left(\lambda_{3} M\right) \operatorname{tr}\left(\lambda_{8}^{\prime} M\right)+\mathcal{K} \operatorname{tr}\left(\lambda_{3} M\right) \operatorname{tr} M\right] \operatorname{tr} P \\
\left.+\left[\mathcal{L} \operatorname{tr}\left(\lambda_{3} M\right) \operatorname{tr}\left(\lambda_{8}^{\prime} M\right)+\mathcal{M} \operatorname{tr}\left(\lambda_{3} M\right) \operatorname{tr} M\right] \operatorname{tr}\left(\lambda_{8}^{\prime} P\right)\right\} \\
\mathcal{F}=\left(2 r_{m}-1\right)\left(b_{P}-b_{A}\right)+6 r_{m}\left(\bar{b}_{P}-\bar{b}_{A}+\bar{d}_{m}\right)+b_{m}=\mathcal{A}+6 \mathcal{B}+18 \mathcal{C} \\
\mathcal{G}=\frac{3}{2}\left(b_{A}+2 b_{m}+2 r_{P} d_{P}\right) \\
\mathcal{H}=3\left(\bar{b}_{P}+\bar{b}_{m}-\bar{b}_{A}\right)+\left(r_{m}+1\right)\left(b_{P}-b_{A}\right)+2 b_{m}=2 \mathcal{A}+3 \mathcal{B} \\
\mathcal{I}=3\left(b_{P}-b_{A}+2 r_{m} d_{m}-b_{m}\right)=3 \mathcal{A}+18 \mathcal{D} \\
\mathcal{J}=2 b_{P}+6 f_{P}+2 r_{P} b_{m}-r_{P} b_{A} \\
\mathcal{K}=6 r_{P}\left(\bar{b}_{m}+\bar{d}_{P}-\bar{b}_{A}\right)+2 r_{m}\left(b_{P}+3 f_{P}\right)+2 r_{P}\left(2 b_{m}+d_{P}-b_{A}\right) \\
\mathcal{L}=b_{m}+r_{P} d_{P}-b_{A} / 2=\mathcal{G} / 3-b_{A} / 2 \\
\mathcal{M}=3\left(\bar{b}_{P}+\bar{b}_{m}-\bar{b}_{A}\right)+\left(b_{P}-b_{A}+2 b_{m}\right)+r_{m} b_{P}=\mathcal{H}+r_{m} b_{A}
\end{aligned}
$$

Thus one can determine, in principle, the eight combinations $\mathcal{F}-\mathcal{M}$. As indicated, however, only three of these are independent of the combinations $\mathcal{A}-\mathcal{D}$ that one can obtain using the flavor off-diagonal two-point Ward identities. Thus one can only determine seven combinations of the ten improvement constants which enter (we exclude $b_{A}$ and $f_{A}$ since we have determined these from the left-hand side of the present identity). A particular choice of these seven combinations, is listed in Table II] To further disentangle the coefficients requires three-point Ward identities, which we consider below.

As noted above, implementation of the flavor-diagonal Ward identity necessarily involves quark-disconnected contractions and thus will be numerically challenging. Thus it is interesting to know how many combinations of quark masses are needed. Are simulations in a $2+1$ flavor theory sufficient, or do all three quarks need to be degenerate? It turns out that a combination of simulations with degenerate masses (taking three or more values) and $2+1$ simulations (with at least two values of the light quark mass differing from each of two choices for the strange quark mass) is sufficient. This allows one to disentangle all the different linear and quadratic mass dependences that appear. 


\section{Three-point axial Ward identities}

We now turn to the enforcement of the chiral transformation properties of bilinear operators. The methodology is standard [1, 6, 7, 8, 22]; what we add here is the generalization to non-degenerate masses in the unquenched theory, and the use of identities involving flavorsinglet components. Contact terms limit what can be extracted with this method, and will need to be understood in detail.

We begin with the simplest example, which is that considered in previous calculations. A non-singlet axial transformation with flavor $(i j)$ is performed in a region of space-time $\mathcal{V}$ which includes a bilinear $\mathcal{O}^{(j k)}$ with $k \neq i$. If $\mathcal{O}^{(j k)}=\bar{\psi}^{j} \Gamma \psi^{k}$, then it is transformed into $\delta \mathcal{O}^{(i k)}=\bar{\psi}^{i} \gamma_{5} \Gamma \psi^{k}$. The identity we enforce on the lattice is thus

$$
\left\langle\left(\delta_{A}^{(i j)} \mathcal{S}\right) \widehat{\mathcal{O}}^{(j k)}(y) J^{(k i)}(z)\right\rangle=\left\langle\widehat{\delta \mathcal{O}}^{(i k)}(y) J^{(k i)}(z)\right\rangle+O\left(a^{2}\right)
$$

where $\delta_{A} \mathcal{S}$ is the improved and normalized lattice form of the formal variation of the continuum action

$$
\left(\delta_{A}^{(i j)} \mathcal{S}\right)=a^{4} \sum_{\mathcal{V}}\left[\left(\widehat{m}_{i}+\widehat{m}_{j}\right) \widehat{P}^{(i j)}-\partial_{\mu} \widehat{A}_{\mu}^{(i j)}\right]
$$

with $\mathcal{V}$ a 4-dimensional subset of the lattice containing $y$ but not the source at $z$. (We do not place a "hat" on $\delta_{A} \mathcal{S}$ so as to avoid overloading the notation.) Note that only quark-connected contractions contribute to this Ward identity.

Actually, as is well known, the identity (69) cannot be satisfied simply by on-shell improvement, since the pseudoscalar density appearing in $\left(\delta_{A}^{(i j)} \mathcal{S}\right)$ comes into contact with $\mathcal{O}^{(j k)}$. Additional off-shell improvement terms are needed, having the same quantum numbers as the product $P^{(i j)} \mathcal{O}^{(j k)}$, and having the appropriate dimension. Since there is an explicit factor of $\widehat{m}_{i}+\widehat{m}_{j}$, the only such term with the right dimension and symmetries is $\delta \mathcal{O}^{(i k)}$. In previous work, we have used a mnemonic for obtaining this contact term, namely that we can off-shell improve the bilinears by introducing an additional "equationsof-motion" operator [7]:

$$
a \bar{\psi}^{j}\left(-\overleftarrow{\not D}+m_{j}\right) \Gamma \psi^{k}+a \bar{\psi}^{j} \Gamma\left(\overrightarrow{\not D}+m_{k}\right) \psi^{k}
$$

While adequate for discussing the Ward identities (69), this is potentially misleading for

two reasons. First, the form implies that contact terms between two operators can be factorized into the contribution of one operator times that of the other. In fact, there is no 
such factorization of contact terms. Second, for the operators we consider below, there are several possible contact terms and the mnemonic cannot be easily generalized. Thus we do not use this mnemonic further in this paper. In fact, all we need to know is that there exist possible contact terms involving the operator $\delta \mathcal{O}^{(i k)}$ and multiplied by $\widehat{m}_{i}+\widehat{m}_{j} \propto \widetilde{m}_{i j}+O(a)$.

A convenient way of using eq. (69) is to take the ratio of the two sides having pulled out unknown mass factors. We first define the improved (but not normalized) variation of the action by

$$
\left(\delta_{A}^{(i j), I} \mathcal{S}\right)=\frac{\left(\delta_{A}^{(i j)} \mathcal{S}\right)}{Z_{A}\left(1+a \bar{b}_{A} \operatorname{tr} M+a b_{A} m_{i j}\right)}=a^{4} \sum_{\mathcal{V}}\left[2 \widetilde{m}_{i j} P^{(i j), I}-\partial_{\mu} A_{\mu}^{(i j), I}\right]
$$

This can be determined by calculating $\widetilde{m}_{i j}$ using eq. (49), since by assumption we know $A_{\mu}^{I}$. The ratio we consider is then

$$
\begin{aligned}
R_{O} & =\frac{\left\langle\left(\delta_{A}^{(i j), I} \mathcal{S}\right) \mathcal{O}^{(j k), I}(y) J^{(k i)}(z)\right\rangle}{\left\langle\delta \mathcal{O}^{(i k), I}(y) J^{(k i)}(z)\right\rangle}, \\
& =\frac{Z_{\delta O}}{Z_{A} Z_{O}}\left[1+a\left(\bar{b}_{\delta O}-\bar{b}_{O}-\bar{b}_{A}\right) \operatorname{tr} M+a\left(b_{\delta O} m_{i k}-b_{O} m_{j k}-b_{A} m_{i j}\right)\right]+a c_{O}^{\prime} \widetilde{m}_{i j}+O\left(a^{2}\right) .
\end{aligned}
$$

Here the contact term is included with an unknown coefficient $c_{O}^{\prime} \cdot{ }^{11}$ Requiring $R_{O}$ to be independent of $y$ and $J$ in the chiral limit determines $c_{V}$ and $c_{T}$, and we assume this has been done, so that we know all the $\mathcal{O}^{(i j), I}$. One also obtains information on the $Z_{O}$, as first noted in Ref. 22]. Away from the chiral limit $R_{O}$ should be automatically independent of $y$ and $J$ up to $O\left(a^{2}\right)$, since there are no additional operators with coefficients to tune. Note that the contact term plays no role in this regard. It has the same operator $\delta \mathcal{O}$ present in both numerator and denominator, and so is independent of $y$ and $J$ by itself.

Thus, for convenient choices of $y$ and $J$, evaluating $R_{O}$ away from the chiral limit allows one to determine one combination of improvement coefficients for each of the three independent linear functions of the quark masses, except that one of these is not useful as it is "contaminated" by the contact term. One complication compared to the quenched case is that the mass dependence of the contact term, $\widetilde{m}_{i j}$, is not simply proportional to $m_{i j}$, but has an additional part proportional to $\left(r_{m}-1\right) \operatorname{tr} M$, as can be seen from eq. (49). Because

${ }^{11}$ Our convention for $c_{O}^{\prime}$ differs from that defined in Ref. [7]. 
of this, a convenient choice of basis is $\widetilde{m}_{i j}, m_{i}-m_{j}$ and $\operatorname{tr} M$, using which we find

$$
\begin{aligned}
R_{O}= & \frac{Z_{\delta O}}{Z_{A} Z_{O}}\left\{1+a\left[\bar{b}_{\delta O}-\bar{b}_{O}-\bar{b}_{A}+\left(b_{\delta O}-b_{O}\right)\left(2+r_{m}\right) / 6+b_{A}\left(r_{m}-1\right) / 3\right] \operatorname{tr} M\right. \\
& \left.\quad+a\left(b_{\delta O}+b_{O}\right)\left(m_{i}-m_{j}\right) / 4\right\}+a\left[c_{O}^{\prime}-\left(b_{\delta O}-b_{O}+2 b_{A}\right) \frac{Z_{\delta O}}{2 Z_{O} Z_{P} Z_{m}}\right] \widetilde{m}_{i j}+O\left(a^{2}\right) .
\end{aligned}
$$

The combinations of the improvement coefficients multiplying $\operatorname{tr} M$ and $m_{i}-m_{j}$ in this equation can, in principle, be determined. To do so it is sufficient to use a $2+1$ flavor theory: the coefficient of $\operatorname{tr} M$ can be determined using $m_{i}=m_{j} \neq m_{k}$, and that of $m_{i}-m_{j}$ can then be determined using $m_{i} \neq m_{j}=m_{k}$. To simply the discussion below, we note that a quick way of determining the accessible combinations of improvement coefficients is to set $\widetilde{m}_{i j}=0$, so that one can make the substitution $m_{j k}=-\operatorname{tr} M\left(r_{m}-1\right) / 3$. The resulting coefficients of $m_{i}-m_{j}$ and $\operatorname{tr} M$ are those that can be determined. ${ }^{12}$

Applying this method to the bilinears in turn, we obtain the results given in Table II] These allow the determination of four new quantities: $Z_{A}, Z_{S} / Z_{P}, b_{T}$ and $\bar{b}_{A}$ (where for the latter we have used knowledge of $b_{A}$ and $r_{m}$ from the two-point Ward identities). The combinations of $S$ and $P$ improvement coefficients that are obtained, however, are all related to those obtained from the two-point Ward identities using the relations listed in Table【. Thus we obtain a check of these relations, but no new information on the constants themselves.

To determine further improvement coefficients we consider axial Ward identities involving the transformation of flavor diagonal bilinears. These have not been considered previously, and, in particular, they are not needed in the quenched approximation. They involve quarkdisconnected contractions in an essential way. The analysis is simplified by the observation that the Ward identities considered above allow the complete determination of $\widehat{A}_{\mu}^{(i j)}$ and thus of the improved variation of the action, $\left(\delta_{A}^{(j k)} \mathcal{S}\right)$, including its normalization. Thus the only unknown coefficients appearing in the three-point axial Ward identities we consider below are those in the operator $\mathcal{O}$ and its axial variation $\delta \mathcal{O}$. We will also make use of the previous determination of $r_{m}$.

\footnotetext{
${ }^{12}$ Note that this is a theoretical device and not a practical tool. Setting $\widetilde{m}_{i j}=0$ and considering $m_{1}-m_{2} \neq 0$ implies that some quark masses are negative. This is undesirable in practice due to the possible phase structure at $m \sim 0$. In practice one would likely need do a fit using positive quark masses in order to separate the different mass dependencies.
} 
We first consider the axial rotation properties of the flavor-singlet operators. The singlet axial current should be invariant under non-singlet axial transformations, so we enforce

$$
\left\langle\left(\delta_{A}^{(12)} \mathcal{S}\right) \widehat{\operatorname{tr} A}_{\mu}(y) J^{(21)}(z)\right\rangle=O\left(a^{2}\right), \quad[j \neq k]
$$

We choose flavor indices 1 and 2 so that we can use the standard Gell-Mann basis of $S U(3)$ matrices - permutations of indices are, of course, allowed. Since the right-hand side of eq. (176) vanishes (at the order we are working), this relation can only determine relative normalizations between independent operators appearing on the left-hand-side. Using eqs. (10) and (25), one finds

$$
\begin{aligned}
\widehat{\operatorname{tr} A_{\mu}} & \propto \operatorname{tr} A_{\mu}+a \bar{c}_{A} \partial_{\mu} \operatorname{tr} P+a d_{A} \operatorname{tr}\left(M A_{\mu}\right) \\
& \propto \operatorname{tr} A_{\mu}+a \bar{c}_{A} \partial_{\mu} \operatorname{tr} P+a\left(d_{A} / 6\right)\left[3 \operatorname{tr}\left(\lambda_{3} M\right) \operatorname{tr}\left(\lambda_{3} A_{\mu}\right)+\operatorname{tr}\left(\lambda_{8}^{\prime} M\right) \operatorname{tr}\left(\lambda_{8}^{\prime} A_{\mu}\right)\right] .
\end{aligned}
$$

In the second line we have used

$$
\operatorname{tr}(M \mathcal{O})=\frac{1}{3} \operatorname{tr}(M) \operatorname{tr}(\mathcal{O})+\frac{1}{2} \operatorname{tr}\left(\lambda_{3} M\right) \operatorname{tr}\left(\lambda_{3} \mathcal{O}\right)+\frac{1}{6} \operatorname{tr}\left(\lambda_{8}^{\prime} M\right) \operatorname{tr}\left(\lambda_{8}^{\prime} \mathcal{O}\right)
$$

valid for diagonal mass matrices, to express the result in terms of independent operators, and absorbed the contribution proportional to $\operatorname{tr} M \operatorname{tr} A_{\mu}$ into the overall constant. In the chiral limit only the first two terms in (77) are present and so $\bar{c}_{A}$ can be determined. Away from the chiral limit, we must avoid contact terms, which are proportional to $\widetilde{m}_{12}$. Following the discussion above, we do so by setting $\widetilde{m}_{12}=0$, leading to $\operatorname{tr}\left(\lambda_{8}^{\prime} M\right)=-2 r_{m} \operatorname{tr} M$, so that eq. (777) becomes

$$
\left.\widehat{\operatorname{tr} A_{\mu}}\right|_{\tilde{m}_{12}=0} \propto \operatorname{tr} A_{\mu}+a \bar{c}_{A} \partial_{\mu} \operatorname{tr} P+a\left(d_{A} / 6\right)\left[3 \operatorname{tr}\left(\lambda_{3} M\right) \operatorname{tr}\left(\lambda_{3} A_{\mu}\right)-2 r_{m} \operatorname{tr} M \operatorname{tr}\left(\lambda_{8}^{\prime} A_{\mu}\right)\right] .
$$

Thus $d_{A}$ can be determined by tuning the cofficients of either $\operatorname{tr}\left(\lambda_{3} A_{\mu}\right)$ or (assuming $r_{m}$ is known) $\operatorname{tr}\left(\lambda_{8}^{\prime} A_{\mu}\right)$. Both require non-degenerate quarks (degenerate quarks are not sufficient since the constraint $\tilde{m}_{12}=0$ then implies $\operatorname{tr}\left(\lambda_{3} M\right)=\operatorname{tr} M=0$ ), but $2+1$ flavors suffice $\left(m_{1}=m_{2} \neq m_{3}\right.$ to determine the coefficient of $\operatorname{tr}\left(\lambda_{8}^{\prime} A_{\mu}\right)$, and $m_{1} \neq m_{2}=m_{3}$ for that of $\left.\operatorname{tr}\left(\lambda_{3} A_{\mu}\right)\right)$. The net result is the first determination of both $\bar{c}_{A}$ and $d_{A}$.

An almost identical discussion holds for the flavor singlet vector bilinear, the conclusion from which is that one can determine $\bar{c}_{V}$ (for the first time) and $d_{V}$ (which checks the determination from the vector charge). 
For the other three bilinears the Ward identities are different, since the singlet bilinears are not invariant:

$$
\begin{aligned}
\left\langle\left(\delta_{A}^{(j k)} \mathcal{S}\right) \widehat{\operatorname{tr} P}(y) J^{(k j)}(z)\right\rangle & =2\left\langle\widehat{S}^{(j k)}(y) J^{(k j)}(z)\right\rangle+O\left(a^{2}\right), \\
\left\langle\left(\delta_{A}^{(j k)} \mathcal{S}\right) \widehat{\operatorname{tr} T}_{\mu \nu}(y) J^{(k j)}(z)\right\rangle & =-\epsilon_{\mu \nu \rho \sigma}\left\langle\widehat{T}_{\rho \sigma}^{(j k)}(y) J^{(k j)}(z)\right\rangle+O\left(a^{2}\right), \\
\left\langle\left(\delta_{A}^{(j k)} \mathcal{S}\right) \widehat{\operatorname{tr} S}(y) J^{(k j)}(z)\right\rangle & =2\left\langle\widehat{P}^{(j k)}(y) J^{(k j)}(z)\right\rangle+O\left(a^{2}\right) .
\end{aligned}
$$

The general strategy to enforce these relations is to take the ratio of the two sides, and require that the result is unity independent of $y$ and $J$. This should also be true independent of quark masses, as long as one avoids contact terms by keeping $\widetilde{m}_{j k}=0$.

Consider first the transformation of the singlet pseudoscalar, (80), and set $j=1, k=2$ for convenience. If we work at $\widetilde{m}_{12}=0$ to avoid contact terms, we find

$$
\begin{aligned}
\widehat{\operatorname{tr} P}=Z_{P} r_{P}[1 & \left.+a\left(\bar{d}_{P}+d_{P} / 3\right) \operatorname{tr} M\right]\left\{\operatorname{tr} P+a g_{P} \operatorname{Tr}\left(F_{\mu \nu} \widetilde{F}_{\mu \nu}\right)\right. \\
& \left.+a d_{P}\left[\operatorname{tr}\left(\lambda_{3} M\right) \operatorname{tr}\left(\lambda_{3} P\right) / 2-a r_{m} \operatorname{tr} M \operatorname{tr}\left(\lambda_{8}^{\prime} P\right) / 3\right]\right\}+O\left(a^{2}\right) .
\end{aligned}
$$

This implies that we can determine $g_{P}$ (in the chiral limit) and $d_{P}$ (in two independent ways as for $d_{A}$ above, both requiring only $2+1$ flavor simulation). Bringing the overall factor to the RHS of (80) we obtain (again, with $\widetilde{m}_{12}=0$ ):

$$
\frac{\widehat{S}^{(12)}}{Z_{P} r_{P}\left[1+a \operatorname{tr} M\left(\bar{d}_{P}+d_{P} / 3\right)\right]}=\frac{Z_{S}}{Z_{P} r_{P}}\left[1+a \operatorname{tr} M\left(\bar{b}_{S}-\bar{d}_{P}-\left(r_{m}-1\right) b_{S} / 3-d_{P} / 3\right)\right] S^{(12)} .
$$

Thus we can determine $Z_{S} /\left(Z_{P} r_{P}\right)$ (which serves as a check) and the combination multiply$\operatorname{ing} \operatorname{tr} M$ (the latter again requiring only $2+1$ flavors).

The Ward identity just discussed gives the first determination of $d_{P}$. This then allows the linear combinations of constants for the masses and pseudoscalar bilinears determined previously (and given in the second section of Table【) to be simplified. In particular, we can now separately determine $b_{P}, f_{P}, b_{m}$ and $d_{m}$ as well as $d_{P}$. This leaves three combinations that cannot yet be disentangled: $\bar{b}_{m}+\bar{d}_{P}, \bar{b}_{P}-\bar{d}_{P}$, and $\bar{b}_{m}-\bar{d}_{m}$. Note that, at this stage, combination multiplying $\operatorname{tr} M$ in eq. (84) does not determine any further coefficients since $\bar{b}_{S}-\bar{d}_{P}=-\left(\bar{b}_{m}+\bar{d}_{P}\right)$.

The same analysis goes through for the tensor bilinear, leading to the first determination of $r_{T}, \bar{c}_{T}, d_{T}$ and $\bar{b}_{T}-\bar{d}_{T}-\left(r_{m}-1\right) b_{T} / 3$. Since we know $b_{T}$ from previous Ward identities, we can extract $\bar{b}_{T}-\bar{d}_{T}$. 
The analysis for the scalar bilinear is more subtle, due to the presence of the identity operator in $\widehat{\operatorname{trS}}$. The net result, however, is as if this identity component was absent, and we find that one can determine the same list as for the pseudoscalar after permuting $P \leftrightarrow S$ : $g_{S}, d_{S}, Z_{P} /\left(Z_{S} r_{S}\right)$ and (using the previously determined $\left.b_{S}\right) \bar{b}_{P}-\bar{d}_{S}$. Of these, only $d_{S}$ is new. ${ }^{13}$ Using the relation $d_{S}=b_{S}+3 \bar{b}_{S}$ from Table I and the previously determined $b_{S}=-2 b_{m}$, we can extract $\bar{b}_{S}=-\bar{b}_{m}$. This allows one to disentangle the remaining linear combinations of $M$ and $P$ improvement coefficients, so that we can determine $\bar{b}_{m}, \bar{d}_{m}, \bar{b}_{P}$ and $\bar{d}_{P}$ separately.

We now return to the identity operator contribution on the LHS of eq. (82). Formally, this contribution vanishes since it it is invariant under axial transformations. However, this fails on the lattice because the variation of the identity operator does not vanish fast enough to overcome the $1 / a^{3}$ divergence in its coefficient:

$$
\left\langle\left(\delta_{A}^{(j k)} \mathcal{S}\right) J^{(k j)}(z)\right\rangle=O\left(a^{2}\right) .
$$

To overcome this one can explicitly subtract the disconnected contribution

$$
\langle\widehat{\operatorname{tr} S}(y)\rangle \times\left\langle\left(\delta_{A}^{(j k)} \mathcal{S}\right) J^{(k j)}(z)\right\rangle
$$

from the LHS of eq. (82). This is equivalent to enforcing the difference of two continuum Ward identities, with coefficients chosen so as to completely remove the contribution from the $e_{S}$ term, including its mass dependence. The cancellation between the two terms is between contributions proportional to $1 / a^{3}$ leaving a residue that must be accurate to $O\left(a^{2}\right) .{ }^{14}$ Thus it will require good statistical control. On the other hand, the dominant contribution to the two terms will be correlated (since it involves the identity operator), which will help the cancellation. ${ }^{15}$

In addition to allowing the separation of all the coefficients for $M$ and $P$, this Ward identity gives a new method for calculating $b_{g}$, using the relation $b_{g}=2 g_{0}^{2} g_{S}$ and the de-

${ }^{13}$ Using the relations in Table 【 $\bar{b}_{P}-\bar{d}_{S}=\left(\bar{b}_{P}-\bar{d}_{P}\right)+\left(\bar{d}_{P}+\bar{b}_{m}\right)+2\left(\bar{d}_{m}-\bar{b}_{m}\right)+2 d_{m} / 3-2 b_{m} / 3$, and all the coefficients appearing on the RHS are known.

${ }^{14}$ Note that all three operators in $\widehat{\operatorname{tr} S}$, i.e. $\operatorname{tr} S, \operatorname{Tr}\left(F_{\mu \nu} F_{\mu \nu}\right)$, and $\operatorname{tr}(M S)$, lead to contributions to $\left.\widehat{\operatorname{tr} S}(y)\right\rangle$ which are separately divergent, but each can be combined with its subtraction term and then multiplied by the unknown coefficient to be determined.

15 This situation is similar to the subtraction of power divergent mixing in weak matrix element calculations in the quenched approximation, which also benefits from correlations between the two quantities being subtracted, and has been successfully carried out in practice $23,24,25]$. 
termination of $g_{S}$. Note that this determination can be carried out in the chiral limit, and so one does not need to know $b_{g}$ a priori. This makes the determination of improvement coefficients using the Ward identities discussed here self contained.

The final Ward identity giving new information is

$$
\left\langle\left(\delta_{A}^{(j k)} \mathcal{S}\right) \widehat{T}_{\mu \nu}^{(k j)}(y) J(z)\right\rangle=-\frac{1}{2} \epsilon_{\mu \nu \rho \sigma}\left\langle\left[\widehat{T}_{\rho \sigma}^{(j j)}(y)+\widehat{T}_{\rho \sigma}^{(k k)}(y)\right] J(z)\right\rangle+O\left(a^{2}\right)
$$

In particular, the RHS contains the diagonal non-singlet, and thus provides access to $f_{T}$ for the first time. To see what we learn from enforcing this identity, we divide through by

$$
Z_{T}\left[1+a \operatorname{tr} M \bar{b}_{T}+a m_{j k} b_{T}\right] \underset{\tilde{m}_{j k}=0}{\longrightarrow} Z_{T}\left[1+a \operatorname{tr} M\left(\bar{b}_{T}-\frac{r_{m}-1}{3} b_{T}\right)\right] .
$$

Then we know the quantities on the LHS of the Ward identity. The operator on the RHS becomes (again setting $j=1$ and $k=2$, and still working at $\tilde{m}_{12}=0$ )

$$
\begin{gathered}
\frac{\widehat{T}_{\rho \sigma}^{(11)}+\widehat{T}_{\rho \sigma}^{(22)}}{Z_{T}\left[1+a \operatorname{tr} M\left(\bar{b}_{T}-\frac{r_{m}-1}{3} b_{T}\right)\right]}=\left(\frac{2 r_{T}}{3}+\mathcal{N} a \operatorname{tr} M\right)\left(\operatorname{tr} T_{\rho \sigma}\right)^{I}+\left(\frac{1}{3}+\mathcal{P} a \operatorname{tr} M\right) \operatorname{tr}\left(\lambda_{8}^{\prime} T_{\rho \sigma}\right)^{I} \\
+\mathcal{Q} a \operatorname{tr}\left(\lambda_{3} M\right) \operatorname{tr}\left(\lambda_{3} T_{\rho \sigma}\right)
\end{gathered}
$$

where

$$
\begin{aligned}
\mathcal{N} & =\frac{2}{3}\left[r_{T}\left(\bar{d}_{T}-\bar{b}_{T}+\frac{d_{T}}{3}+\frac{r_{m}-1}{3} b_{T}\right)-r_{m}\left(f_{T}+\frac{b_{T}}{3}\right)\right], \\
\mathcal{P} & =\frac{2}{9} r_{m}\left(b_{T}-r_{T} d_{T}\right) \\
\mathcal{Q} & =\frac{1}{6}\left(b_{T}+2 r_{T} d_{T}\right) .
\end{aligned}
$$

Thus in the chiral limit we can determine $r_{T}, \bar{c}_{T}$ and $c_{T}$, which provide cross-checks, while away from the chiral limit we obtain the three combinations $\mathcal{N}, \mathcal{P}$ and $\mathcal{Q}$ (all obtainable with $2+1$ flavors). These in turn can be combined to give $b_{T}$ and $d_{T}$ separately, as well as the quantity $r_{T}\left(\bar{d}_{T}-\bar{b}_{T}\right)-r_{m} f_{T}$. Given the determination of $\bar{d}_{T}-\bar{b}_{T}$ from the Ward identity (81) above, we can extract $f_{T}$. We cannot, however, see any way of disentangling $\bar{d}_{T}$ and $\bar{b}_{T}$.

Although we have avoided contact terms by working at $\widetilde{m}_{12}=0$, we note that these terms are more complicated here than in the previous Ward identity. The contact terms arise from the fact that, even if one has on-shell improved the operator $\widehat{P}^{(j k)}$ appearing in $\left(\delta_{A}^{(j k)} \mathcal{S}\right)$ and the tensor bilinear $\widehat{T}_{\mu \nu}^{(k j)}$, the product of these operators at the same position 
will not be improved. To improve this product one needs to add all operators with the same transformation properties as the product, and having appropriate dimension (here dimension 4 because of the overall factor of $\widehat{m}_{j}+\widehat{m}_{k}$ ). In the Ward identity considered above, in which the bilinear on the RHS was flavor off-diagonal, only a single operator could appear, namely $\delta \mathcal{O}$ with appropriate flavor indices. By contrast, in the present identity, two operators are allowed, namely those appearing on the RHS,

$$
a \widetilde{m}_{j k}\left(\delta T_{\mu \nu}^{(j j)}(y)+\delta T_{\mu \nu}^{(k k)}(y)\right) \quad \text { and } \quad a \widetilde{m}_{j k} \operatorname{tr}\left(\delta T_{\mu \nu}\right)(y)
$$

The latter arises from Wick contractions in which the quark and antiquark in $P^{(j k)}$ are both contracted with the corresponding antiquark and quark in $T^{(k j)}$. To understand (93) in terms of operators vanishing by the equations of motion requires a generalization of the prescription given in Ref. [7]. The appearance of a second operator plays an important role in the discussion of vector Ward identities in appendix C

The remaining Ward identities do not provide any new information on the improvement coefficients, but do provide several important cross-checks. Consider first

$$
\left\langle\left(\delta_{A}^{(j k)} \mathcal{S}\right) \widehat{S}^{(k j)}(y) J(z)\right\rangle=\left\langle\left[\widehat{P}^{(j j)}(y)+\widehat{P}^{(k k)}(y)\right] J(z)\right\rangle+O\left(a^{2}\right)
$$

Dividing both sides by

$$
Z_{S}\left[1+a \operatorname{tr} M\left(\bar{b}_{S}-\frac{r_{m}-1}{3} b_{S}\right)\right]
$$

the LHS is then a known quantity, while the RHS becomes (picking $j=1, k=2$ and setting $\left.\widetilde{m}_{12}=0\right)$

$$
\begin{gathered}
\frac{\widehat{P}^{(11)}+\widehat{P}^{(22)}}{Z_{S}\left[1+a \operatorname{tr} M\left(\bar{b}_{S}-\frac{r_{m}-1}{3} b_{S}\right)\right]}=\frac{Z_{P}}{Z_{S}}\left\{\left(\frac{2 r_{P}}{3}\right.\right. \\
\left.+\mathcal{N}^{\prime} a \operatorname{tr} M\right)(\operatorname{tr} P)^{I}+\left(\frac{1}{3}+\mathcal{P}^{\prime} a \operatorname{tr} M\right) \operatorname{tr}\left(\lambda_{8}^{\prime} P\right)^{I} \\
\left.+\mathcal{Q}^{\prime} a \operatorname{tr}\left(\lambda_{3} M\right) \operatorname{tr}\left(\lambda_{3} P\right)\right\}
\end{gathered}
$$

where

$$
\begin{aligned}
\mathcal{N}^{\prime} & =\frac{2}{3}\left[r_{P}\left(\bar{d}_{P}-\bar{b}_{S}+\frac{d_{P}}{3}+\frac{r_{m}-1}{3} b_{S}\right)-r_{m}\left(f_{P}+\frac{b_{P}}{3}\right)\right], \\
\mathcal{P}^{\prime} & =\frac{1}{9}\left[3\left(\bar{b}_{P}-\bar{b}_{S}\right)+b_{P}-b_{S}+r_{m}\left(b_{P}+b_{S}-2 r_{P} d_{P}\right)\right], \\
\mathcal{Q}^{\prime} & =\frac{1}{6}\left(b_{P}+2 r_{P} d_{P}\right) .
\end{aligned}
$$


A similar analysis holds for the Ward identity with $S$ and $P$ interchanged, except that one must subtract the disconnected component from both sides of the equation. Combining these two Ward identities allows the determination of the coefficients listed in Table II

In fact, the disconnected component on the LHS of the Ward identity (94) with $S$ and $P$ interchanged gives access to the quark condensate:

$$
\frac{1}{Z_{P}}\left\langle\left(\delta_{A}^{(j k)} \mathcal{S}\right) \widehat{P}^{(k j)}(y)\right\rangle \equiv \frac{1}{Z_{P}}\left\langle\widehat{S}^{(j j)}(y)+\widehat{S}^{(k k)}(y)\right\rangle+O\left(a^{2}\right) \quad \widetilde{m}_{j k}=0 .
$$

Here the idea is to calculate the LHS, and use it as the definition of the RHS. We know all the improvement and renormalization constants appearing on the LHS, and, by working at $\widetilde{m}_{j k}=0$ we avoid contact terms. In this way we can calculate the improved condensate, including its dependence on $\widehat{m}_{l}$. This is the $O(a)$ improved version of the method first suggested in Ref. [22].

The final two Ward identities of this type involve vector and axial currents, e.g.

$$
\left\langle\left(\delta_{A}^{(j k)} \mathcal{S}\right) \widehat{A}_{\mu}^{(k j)}(y) J(z)\right\rangle=\left\langle\left[\widehat{V}_{\mu}^{(k k)}(y)-\widehat{V}_{\mu}^{(j j)}(y)\right] J(z)\right\rangle+O\left(a^{2}\right) .
$$

Now only flavor non-singlet operators appear, so we cannot obtain information about singlet improvement coefficients. In fact, this identity and that with $V \leftrightarrow A$ only differ from the previous non-singlet axial Ward identities, eq. (69), when $m_{j} \neq m_{k}$. In this case there are additional quark disconnected contractions absent in (69). Since we are assuming that we know the improved off-diagonal axial current, the LHS of this relation is completely known, allowing a complete determination of the constants appearing on the RHS. It is a straightforward exercise using the form of the improved diagonal non-singlet bilinears,

eq. (18), to show that the new information that one obtains here over that obtained using eq. (69), is a direct determination of $f_{V}$ and $b_{V}$. In particular, this Ward identity provides the only cross check of the calculation of $f_{V}$. The identity with $V \leftrightarrow A$ similarly provides an alternative determination of $f_{A}$.

\section{SUMMARY AND CONCLUSIONS}

Improvement in the presence of non-degenerate dynamical quarks is far more complicated than that with degenerate quarks or that in the quenched approximation. Nevertheless, the considerable number of extra improvement coefficients that arise for quark bilinears and 
quark masses can almost all be determined by enforcing the vector and axial transformation properties of these operators. The results from the Ward identities considered above are collected in Tables 【 and II The only scale independent quantities which are left undetermined are $\bar{d}_{A}$ and $\bar{b}_{T}+\bar{d}_{T}{ }^{16}$ To determine these one must use other methods. The only two that we are aware of are improved non-perturbative renormalization [10, 11], and matching to perturbative forms for short distance correlation functions of bilinears [8]. The scale-dependent quantitites $Z_{T}, Z_{S} Z_{P}$, and $r_{A}$ are also undetermined, but this had to be the case as Ward identities do not involve a renormalization scale. For these one must use a method like non-perturbative renormalization.

It is interesting to understand why the two scale independent quantities cannot be determined using Ward identities. In the case of $\bar{d}_{A}$, the reason is the lack of an identity involving $\operatorname{tr} A_{\mu}$ that has a non-vanishing variation. Thus the overall normalization factor, which includes $\bar{d}_{A}$, cannot be determined. This is clearly related to the fact that $r_{A}$ cannot be determined, because it is scale-dependent.

The reason is similar for $\bar{b}_{T}+\bar{d}_{T}$. Ward identities relate the flavor singlet tensor to the flavor non-singlet tensor. Overall factors thus cancel, and the mass dependent part of the overall factor is proportional to $\bar{b}_{T}+\bar{d}_{T}$. Thus, in essence, this combination cannot be determined because $Z_{T}$ cannot.

To test these arguments, and for completeness, we have extended the analysis to two and four non-degenerate flavors. These cases are also of phenomenological interest. A summary of the results is given in appendices $\mathrm{A}$ and $\mathrm{B}$ With four flavors, one might naively have hoped to determine more coefficients, since the three flavor theory is included as a subset. We find, however, that although most Ward identities by themselves allow more combinations of coefficients to be determined, so that the analysis is cleaner, the final result is the same as with three flavors. In particular, $\bar{d}_{A}$ and $\bar{b}_{T}+\bar{d}_{T}$ cannot be determined, for exactly the same reasons as for three flavors.

With two flavors, there are, on the one hand, fewer coefficients to determine, but, on the other hand, fewer Ward identities. Furthermore, each identity determines fewer coefficients because there are less masses to vary independently. The net result is that there are more undetermined combinations of scale independent quantities than for three or four flavors

$\overline{16}$ This corrects the conclusion of Ref. 12] that there were three undetermined scale independent quantities. 
(eight in all). Particularly striking is the result that one cannot determine the improved flavor non-singlet axial current, pseudoscalar density or tensor bilinear away from the chiral limit.

Given the complexity of the calculations we outline, one might wonder about possible simplifications. Since adding flavors extends $\left(N_{f}=2 \rightarrow 3\right)$ or simplifies $\left(N_{f}=3 \rightarrow 4\right)$ the analysis, one might consider using a partially quenched simulation with, say $2+1$ flavors of sea quarks, and four or more flavors of valence quarks. To add additional information over the unquenched analysis, one must necessarily consider theories with differing sea and valence content, which are therefore not unitary. This makes the basis of the Symanzik improvement program less secure. Nevertheless, it is certainly possible, assuming the improvement program remains valid, to use the enlarged graded symmetry groups of partially quenched theories to constrain the allowed improvement coefficients, and to generalize the analysis presented here.

Staying within the unquenched three flavor theory, one can ask whether it is sufficient to use $2+1$ flavor theories in which at least two quarks are degenerate. This point has been discussed for each identity considered in the text, and we find that $2+1$ flavors is sufficient to determine all the allowed coefficients.

Another practical question is whether one needs to determine all the improvement coefficients for phenomenologically interesting applications. Particularly interesting are (1) matrix elements of the electromagnetic current, (2) matrix elements of flavor off-diagonal vector and axial currents (for weak transitions), (3) quark masses and (4) matrix element of the mass term in the action, $m_{j} S^{(j j)}$. We consider these cases in turn. (1) The improvement and renormalization coefficients for the electromagnetic current, which is a flavor non-singlet for $N_{f}=3$, are $Z_{V}, b_{V}, \bar{b}_{V}$ and $f_{V}$, and these can all be determined by normalizing the matrix elements of the charge. The determination does, however, require quark-disconnected matrix elements. This also determines the improved flavor off-diagonal vector current. (2) The off-diaogonal axial current requires $Z_{A}, b_{A}$ and $\bar{b}_{A}$, which can all be determined from flavor off-diagonal three point Ward identities, as long as we know $b_{V}$. Thus only quarkconnected contractions are needed. (3) Improvement of individual quark masses requires $Z_{m}, r_{m}, b_{m}, \bar{b}_{m}, d_{m}$ and $\bar{d}_{m}$, i.e. both flavor singlet and non-singlet coefficients [see eq. (26)]. To determine these requires all the types of Ward identity we consider, i.e. two and threepoint Ward identities involving both flavor singlet and non-singlet operators. The same is 
true for (4), the combination $m_{j} S^{(j j)}$. Thus we see that for some applications there are considerable simplifications, but for others there are not. We stress however, that for all of these quantities one must first determine $b_{g}$, although, as discussed in the introduction, a perturbative determination of this numerically small coefficient may be sufficient.

Finally, we note that one might consider our work as an advertisement for other approaches to improvement with Wilson-like fermions, namely "Wilson averaging" [26] and twisted mass QCD 27] at maximal twist [26]. In both approaches the $O(a)$ terms are automatically absent in the physical matrix elements of the operators we consider here, so that no improvement of the operators themselves is necessary. For this to hold, however, one needs an even number of fermions, and so for the physical case one must simulate with four flavors. First work in this direction has begun [28].

\section{Acknowledgments}

We thank Rainer Sommer for comments on the manuscript. This work was supported in part by US Department of Energy through grants DE-FG03-96ER40956/A006 and KA-0401010-E161, and by the BK21 program at Seoul National University, the SNU foundation \& Overhead Research fund and the Korea Research Foundation through grant KRF-2002003-C00033.

\section{APPENDIX A: FOUR NON-DEGENERATE FLAVORS}

In this section we briefly describe the generalization of our calculations to $N_{f}=4$. Having one additional flavor does not change the number of improvement coefficients, but does increase the number of independent masses that one can vary. This allows the extraction of additional improvement coefficients in many of the Ward identities. The final result, however, is the same as for $N_{f}=3$ : all scale independent coefficients can be determined except for $\bar{d}_{A}$ and $\bar{b}_{T}+\bar{d}_{T}$.

We summarize the results in Tables \and III and in the following comment on the ways in which the calculation differs from that with $N_{f}=3$.

1. The general forms for the improvement of bilinears and masses, eqs. (15 232425), are unchanged, except that $\lambda$ are now $S U(4)$ generators. This is because no special 
TABLE III: Normalization and improvement coefficients determined using various Ward identities (which are denoted schematically) for $N_{f}=4$. Notation as in Table II

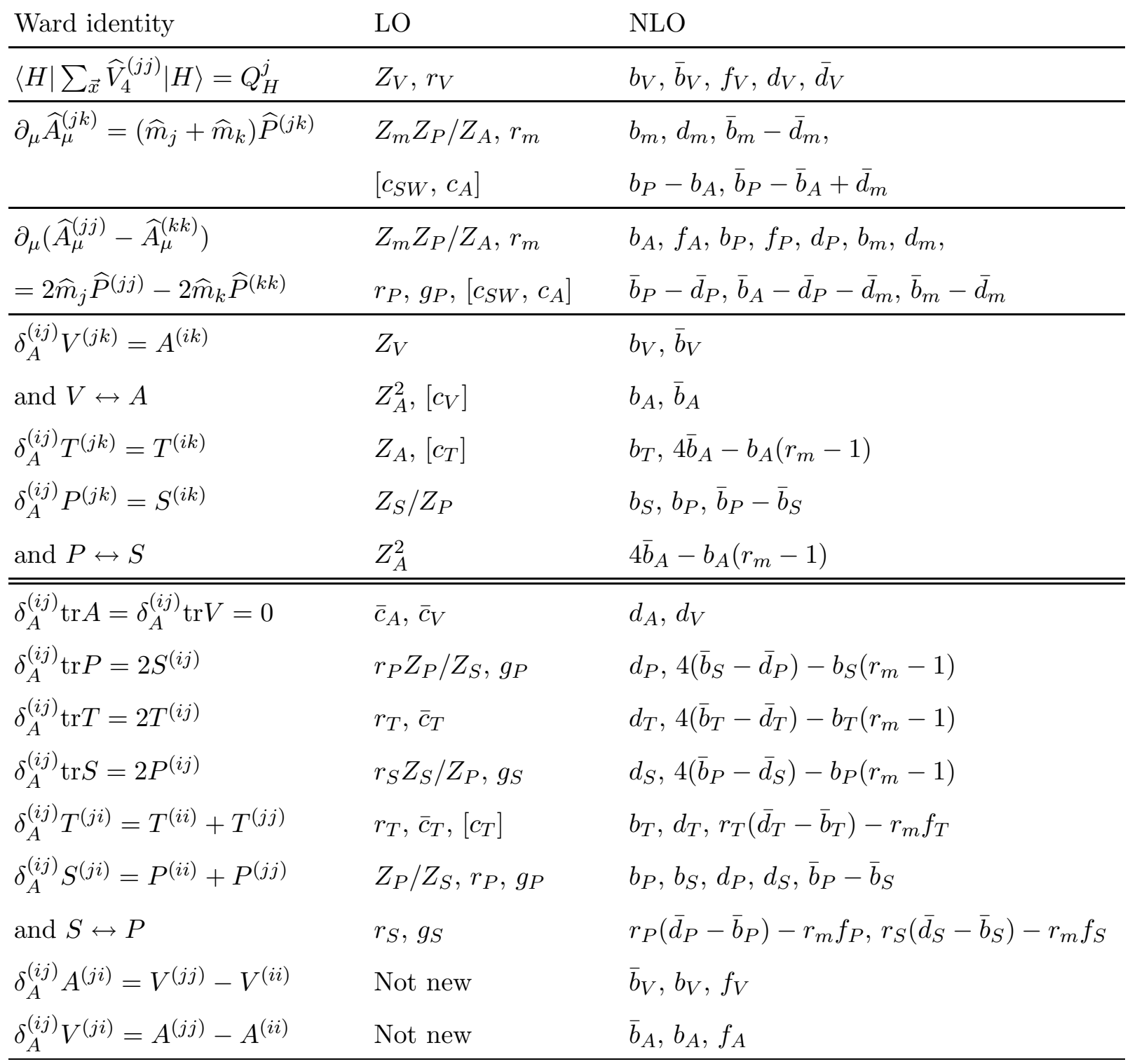

properties of $S U(3)$ have been used in writing these equations. Thus the number of improvement coefficients is unchanged from $N_{f}=3$.

2. The use of the vector Ward identity, eq. (27), is also unchanged.

3. The enforcement of " $Z_{S} Z_{m}=1$ ", i.e. eq. (33), is similar to the three flavor case, although more complicated algebraically as it involves determinants of $4 \times 4$ matrices. The results are given in Table I some are identical to those with $N_{f}=3$, while others 
have $N_{f}$ dependence. There remains a single constraint, due to the absence of an "f-term" in the improvement of the quark masses.

4. The two-point axial Ward identities are somewhat more powerful than for $N_{f}=3$, due to the greater number of independent combinations of masses that can be constructed, particularly at quadratic order. As can be seen from Table III, one can determine ten combinations of improvement coefficients, all of which are fairly simple, compared to nine when $N_{f}=3$, some of which are complicated (see Table II). For four flavors the flavor off-diagonal identity, eq. (47), becomes redundant, giving no information not contained in the flavor diagonal identity (54).

5. Three-point Ward identities involving only off-diagonal bilinears, eq. (69), are also more powerful, because there is one additional combination of masses that is independent from that multiplying the contact term. This allows the separate determination of the $b_{O}$ from these identities alone, and of $\bar{b}_{A}, \bar{b}_{V}$, and $\bar{b}_{S}-\bar{b}_{P}$. On the other hand, the net result, including information from previous identities, is the same as for $N_{f}=3$ : the newly determined constants remain $Z_{A}, Z_{S} / Z_{P}, \bar{b}_{A}$ and $b_{T}$.

6. The Ward identities involving axial transformation of flavor singlets, e.g. eq. (76), give essentially the same information as for $N_{f}=3$. The extra combination of masses that is available does not multiply new combinations of coefficients. These identities continue to provide the only determination of $d_{A}, \bar{c}_{A}$ and $\bar{c}_{V}$, as well as the first determination of $d_{S}$. The latter, together with the constraint $d_{S}=b_{S}+N_{f} \bar{b}_{S}$, allows the separate determination of all the mass and pseudoscalar improvement coefficients, as for $N_{f}=3$.

7. The final type of identity, exemplified by eq. (87), is more powerful with $N_{f}=4$, as can be seen from the tables. Nevertheless, the only new information obtained is $f_{T}$.

In summary, with four flavors one has considerably more cross checks, and the extraction of individual improvement coefficients is more straightforward, but in the end one obtains the same set of coefficients as for three flavors. 


\section{APPENDIX B: TWO NON-DEGENERATE FLAVORS}

In this appendix we describe how our considerations change when $N_{f}=2$. The first new feature is that there is one less independent improvement coefficient for each bilinear and for the quark masses. This follows from the fact that $d_{a b c} \propto \operatorname{Tr}\left(\sigma_{a}\left\{\sigma_{b}, \sigma_{c}\right\}\right)=0$ in $S U(2)$ (with $\sigma_{a}, a=1,2,3$ the Pauli matrices). Thus one has

$$
\begin{gathered}
\left\{\sigma_{a}, M\right\}=\sigma_{a} \operatorname{tr} M+\operatorname{tr}\left(\sigma_{a} M\right) \mathbb{1} \Longrightarrow(1) \operatorname{tr}\left(\left\{\sigma_{a}, M\right\} \mathcal{O}\right)=\operatorname{tr} M \operatorname{tr}\left(\sigma_{a} \mathcal{O}\right)+\operatorname{tr}\left(\sigma_{a} M\right) \operatorname{tr} \mathcal{O} \\
(2) \operatorname{tr}\left(\sigma_{a} M^{2}\right)=\operatorname{tr}\left(\sigma_{a} M\right) \operatorname{tr} M
\end{gathered}
$$

This means that the $b_{O}$ term in eq. (15), and the $b_{m}$ term in eq. (24), are not independent and can be absorbed into the other terms by changing their coefficients as follows:

$$
\bar{b}_{\mathcal{O}} \longrightarrow b_{\mathcal{O}}^{\prime}=\bar{b}_{\mathcal{O}}+b_{\mathcal{O}} / 2, \quad \bar{f}_{\mathcal{O}} \longrightarrow f_{\mathcal{O}}^{\prime}=f_{\mathcal{O}}+b_{\mathcal{O}} / 2, \quad \bar{b}_{m} \longrightarrow \bar{b}_{m}^{\prime}=\bar{b}_{m}+b_{m}
$$

In the following, we assume that this has been done, and that the primes are then dropped.

Although there are thus six less coefficients to determine, it turns out that there are fewer Ward identities available, and that each is less powerful than for $N_{f}=3$. The results are collected in Tables \and IV and we discuss the salient features in the following.

1. The use of the vector Ward identity, eq. (27), is unchanged, aside from the fact that there is one less coefficient to determine.

2. The enforcement of " $Z_{S} Z_{m}=1$ ", i.e. eq. (33), follows the same steps as above, but leads to simpler relations because of the absence of $b_{m}$ and $b_{S}$. The results in Table remain valid for $N_{f}=2$ as long as one sets $b_{m}=b_{S}=0 .{ }^{17}$

3. The two-point axial Ward identities are less powerful than for $N_{f}=3$, due to the smaller number of independent combinations of masses. Combining the flavor diagonal and off-diagonal identities, one only determines $r_{m} Z_{m} Z_{P} / Z_{A}, r_{P} / r_{m}$ and $g_{P}$ at LO, and $f_{A}, d_{m}, d_{P}$, and the combinations $\bar{d}_{m}+\bar{b}_{P}-\bar{b}_{A}$ and $r_{P}\left(\bar{b}_{m}-\bar{b}_{A}+\bar{d}_{P}\right)+r_{m} f_{P}$ at NLO. Note that one cannot determine $r_{m}$ and $r_{P}$ separately, nor obtain $b_{A}$, unlike for $N_{f}=3$.

\footnotetext{
${ }^{17}$ Alternatively, one can keep the relations as they stand in the Table and then absorb $b_{m}$ and $b_{S}$ into the other coefficients as in eq. (B2).
} 
TABLE IV: Normalization and improvement coefficients determined using various Ward identities (which are denoted schematically) for $N_{f}=2$. Notation as in Table II

\begin{tabular}{lll} 
Ward identity & $\mathrm{LO}$ & $\mathrm{NLO}$ \\
\hline$\left\langle H\left|\sum_{\vec{x}} \widehat{V}_{4}^{(j j)}\right| H\right\rangle=Q_{H}^{j}$ & $Z_{V}, r_{V}$ & $\bar{b}_{V}, f_{V}, d_{V}, \bar{d}_{V}$ \\
\hline$\partial_{\mu} \widehat{A}_{\mu}^{(12)}=\left(\widehat{m}_{1}+\widehat{m}_{2}\right) \widehat{P}^{(12)}$ & $r_{m} Z_{m} Z_{P} / Z_{A},\left[c_{S W}, c_{A}\right]$ & $d_{m}, \bar{b}_{A}-\bar{b}_{P}-\bar{d}_{m}$ \\
\hline$\partial_{\mu}\left(\widehat{A}_{\mu}^{(11)}-\widehat{A}_{\mu}^{(22)}\right)$ & $r_{m} Z_{m} Z_{P} / Z_{A}$ & $f_{A}, r_{P} d_{P}+r_{m} d_{m}$ \\
$=2 \widehat{m}_{j} \widehat{P}^{(11)}-2 \widehat{m}_{k} \widehat{P}^{(22)}$ & $r_{m} / r_{P}, g_{P}$ & $r_{m}\left(d_{m}+2 \bar{d}_{m}+2 \bar{b}_{P}-2 \bar{b}_{A}\right)$ \\
& {$\left[c_{S W}, c_{A}\right]$} & $r_{P}\left(2 \bar{b}_{m}+2 \bar{d}_{P}-2 \bar{b}_{A}+d_{P}\right)+2 r_{m} f_{P}$ \\
\hline$\delta_{A}^{(12)} \operatorname{tr} A=\delta_{A}^{(12)} \operatorname{tr} V=0$ & $\bar{c}_{A}, \bar{c}_{V}$ & $d_{A}, d_{V}$ \\
$\delta_{A}^{(12)} \operatorname{tr} P=2 S^{(12)}$ & $r_{P} Z_{P} Z_{A} / Z_{S}, g_{P}$ & $d_{P}$ \\
$\delta_{A}^{(12)} \operatorname{tr} T=2 T^{(12)}$ & $r_{T} Z_{A}, \bar{c}_{T}, c_{T}$ & $d_{T}$ \\
$\delta_{A}^{(12)} \operatorname{tr} S=2 P^{(12)}$ & $r_{S} Z_{S} Z_{A} / Z_{P}, g_{S}$ & $d_{S}$ \\
$\delta_{A}^{(12)} T^{(21)}=T^{(11)}+T^{(22)}$ & $Z_{A} / r_{T}, \bar{c}_{T}, c_{T}$ & $d_{T}$ \\
$\delta_{A}^{(12)} S^{(21)}=P^{(11)}+P^{(22)}$ & $r_{P} Z_{P} /\left(Z_{A} Z_{S}\right), g_{P}$ & $d_{P}$ \\
$\delta_{A}^{(12)} P^{(21)}=S^{(11)}+S^{(22)}$ & $r_{S} Z_{S} /\left(Z_{A} Z_{P}\right), g_{S}$ & $d_{S}$ \\
$\delta_{A}^{(12)} A^{(21)}=V^{(22)}-V^{(11)}$ & $Z_{V} / Z_{A}^{2}, c_{V},\left[c_{A}\right]$ & $f_{V}$ \\
$\delta_{A}^{(12)} V^{(21)}=A^{(22)}-A^{(11)}$ & $Z_{V}, c_{V},\left[c_{A}\right]$ & $f_{A}$ \\
\hline
\end{tabular}

4. For two flavors there are no three point Ward identities involving only off-diagonal bilinears. Thus one loses what has been one of the central tools in quenched studies. In particular, for $N_{f}=3$ and 4 these are the identities that are used to determine $c_{V}$ and $c_{T}$. Here we need to use other identities for this purpose, as discussed below and indicated in the Table.

5. The Ward identities involving axial transformations of the singlet axial and vector currents, e.g. eq. (76), give the same information as for $N_{f}=3$. However, those involving the tensor, scalar and pseudoscalar bilinears give less information. This is because there is one less combination of quark masses that is independent of the contact term. In fact the analysis is more straightforward, because the contact term is proportional to $\widetilde{m}_{12} \propto \operatorname{tr}(M)+O(a)$, and so one does not need to know $r_{m}$ in order 
to work at $\widetilde{m}_{12}=0$.

At this stage we still have not determined $Z_{A}$ or $\bar{b}_{A}$, so we do not know the normalization of the axial variation of the action. Note also that the tensor Ward identity allows the first determination of $c_{T}$.

6. The final type of identity is that exemplified by eq. (87). This again is simpler to analyze for $N_{f}=2$, since the RHS is a pure flavor singlet (for $T, S$ and $P$ ), rather than a mix of singlet and non-singlet. In particular, new information is obtained in the chiral limit, and allows one, combined with previous results, to disentangle $Z_{A}$ and $r_{T}$.

The identities involving the vector bilinears are the first to allow a determination of $c_{V}$. In fact, in the chiral limit, where one works to determine $c_{V}$, these identities involve the same quark contractions as the three-point Ward identities with only off-diagonal bilinears that are present for $N_{f} \geq 3$. Thus, from a computational point of view, there is no difference in the method to be used to determine $c_{V}$ for $N_{f}=2$. This is not, however, the case for $c_{T}$.

The following scale independent constants remain undetermined by the Ward identities: $\bar{b}_{A}$ and $\bar{d}_{A} ; \bar{b}_{T}, \bar{d}_{T}$ and $f_{T} ; Z_{S} / Z_{P}, r_{P}, \bar{b}_{P}, \bar{d}_{P}$ and $f_{P}$; and $r_{S}$ and $\bar{d}_{S}$ or equivalently $r_{m}$ and $\bar{d}_{m}$, although the following combinations of these coefficients are known:

$$
\bar{b}_{A}-\bar{b}_{P}-\bar{d}_{m}, \quad r_{P}\left(\bar{b}_{A}-\bar{b}_{m}-\bar{d}_{P}\right)-r_{m} f_{P}, \quad r_{P} Z_{P} / Z_{S}, \quad r_{P} r_{S}
$$

Thus in total there are eight undetermined combinations of scale independent coefficients. This is six more than for $N_{f}=3$, despite the need to determine six fewer coefficients.

What is perhaps most striking about this list is that, even if one uses non-degenerate quarks when implementing Ward identities, one cannot determine all the coefficients needed for flavor non-singlet bilinears composed of degenerate quarks. In particular, since one cannot separately determine $\bar{b}_{A}, \bar{b}_{P}$ or $\bar{b}_{T}$, one does not know the overall normalization of the corresponding bilinears away from the chiral limit. To determine this one must use a method such as those proposed in Ref. [8, 11]. 


\section{APPENDIX C: VECTOR WARD IDENTITIES}

In this appendix we explain the claim made in the text that, other than the normalization of charges, enforcing vector Ward identities leads to no information on improvement coefficients. It is interesting to see how this works in detail, and how we concluded otherwise in Ref. [12]. It also gives a good example of how the mnemonic introduced in Ref. 7, 8] of using equations-of-motion operators to determine the form of contact terms needs to be modified.

We begin by recalling some results from the main text. Enforcing the charge of hadrons, eq. (27), determines the improved, renormalized diagonal vector currents, $\widehat{V}_{\mu}^{(j j)}$, aside from the $c_{V}$ and $\bar{c}_{V}$ terms. The latter terms do not contribute to the divergence, so $\partial_{\mu} \widehat{V}_{\mu}^{(j j)}$ is

fully improved. This implies that the divergence of the off-diagonal currents, $\partial_{\mu} \widehat{V}_{\mu}^{(j k)}, j \neq k$, are also improved, because these involve the same improvement coefficients as the diagonal currents (in particular, $Z_{V}, r_{V}, b_{V}$ and $\bar{b}_{V}$; the coefficient $f_{V}$ is known but not required). These results hold whatever the precise form of the underlying lattice current.

Next we recall the form of the exact lattice vector Ward identities. Making the change of variables $\delta_{V} \psi_{j}=\psi_{k}$ and $\delta_{V} \bar{\psi}_{k}=-\bar{\psi}_{j}$ over a region of the lattice $\mathcal{V}$, one finds

$$
\left\langle a^{4} \sum_{\mathcal{V}}\left[\left(m_{j}-m_{k}\right) S^{(j k)}-\partial_{\mu} V_{\mu}^{l a t,(j k)}\right] \mathcal{O}(y) J(z)\right\rangle=\left\langle\left[\delta_{V}^{l a t} \mathcal{O}\right](y) J(z)\right\rangle,
$$

Here $y$ is in the region $\mathcal{V}$, while $z$ is not, and $\delta_{V}^{\text {lat }} \mathcal{O}$ is the variation of the operator $\mathcal{O}$ under the vector transformation. Note that the bare lattice quark masses appear in (C1) irrespective of the presence of the $c_{S W}$ term, and that $S^{(j k)}$ is the local scalar bilinear. The current $V_{\mu}^{l a t,(j k)}$ is the usual lattice vector current, also unaffected by the $c_{S W}$ term. It is associated with a link, and not a site, but its divergence is associated with a site:

$$
\begin{aligned}
V_{\mu}^{l a t,(j k)}\left(x+\frac{\widehat{\mu}}{2}\right) & \equiv \frac{1}{2}\left[\bar{\psi}_{j}(x) \gamma_{\mu} U_{x, \mu} \psi_{k}(x+\widehat{\mu})+\bar{\psi}_{j}(x+\widehat{\mu}) \gamma_{\mu} U_{x, \mu}^{\dagger} \psi_{k}(x)\right], \\
{\left[\partial_{\mu} V_{\mu}^{l a t,(j k)}\right](x) } & \equiv \sum_{\mu} \frac{1}{a}\left[V_{\mu}^{l a t,(j k)}\left(x+\frac{\widehat{\mu}}{2}\right)-V_{\mu}^{l a t,(j k)}\left(x-\frac{\widehat{\mu}}{2}\right)\right]
\end{aligned}
$$

The results (C1) and (C2) hold both for $j \neq k$ and $j=k$. In particular, the diagonal lattice current is conserved, and it generates canonically normalized vector transformations. This implies that the charge constructed from the lattice current is correctly normalized [as can be derived from eq. (C1)]. In other words, for this current, $Z_{V}^{\text {lat }}=1=r_{V}^{\text {lat }}$, and 
the mass dependent improvement coefficients all vanish. As is well known, however, the lattice current is not improved. For this one must add $c_{V^{-}}$and $\bar{c}_{V}$-like terms, as in eq. (6), except that they must be associated with a link, rather than a site. It is possible to do so in such a way that taking the divergence of the current [in the form that appears in the Ward identity ([C1)] exactly cancels the $c_{V^{-}}$and $\bar{c}_{V^{-}}$-like terms. ${ }^{18}$ Because of this the $c_{V^{-}}$and $\bar{c}_{V}$-like terms neither contribute to the vector Ward identities nor affect the values of the other improvement and normalization constants.

In summary, the divergences of the flavor-diagonal conserved lattice vector currents are automatically improved. As noted above, this also implies that the divergences of the flavor off-diagonal lattice vector currents are improved. Thus the $\partial_{\mu} V_{\mu}^{l a t,(j k)}$ term in the vector variation of the action in the lattice Ward identity (C1) is improved. This means that it can be replaced, up to errors of $O\left(a^{2}\right)$, with $\partial_{\mu} \widehat{V}_{\mu}^{(j k)}$, the divergence of the improved local vector current. In this replacement we do not have to worry about contact terms as $\sum_{\mathcal{V}} \partial_{\mu} \widehat{V}_{\mu}$ vanishes except at the surface of the region $\mathcal{V}$, and by assumption there are no other operators there.

The other term in the vector variation of the lattice action in (C1) involves bare masses and scalar densities. As shown by eq. (46), this maintains the same form when written in terms of improved masses and scalar densities.

Combining these observations we can rewrite the lattice vector Ward identity in terms of the improved bilinears and masses considered in the main text:

$$
\left\langle a^{4} \sum_{\mathcal{V}}\left[\left(\widehat{m}_{j}-\widehat{m}_{k}\right) \widehat{S}^{(j k)}-\partial_{\mu} \widehat{V}_{\mu}^{(j k)}\right] \mathcal{O}(y) J(z)\right\rangle=\left\langle\left[\delta_{V}^{l a t} \mathcal{O}\right](y) J(z)\right\rangle+O\left(a^{2}\right),
$$

This relation leads, amongst other things, to the normalization condition for the vector charge, eq. (27). Note that this relation is "off-shell" improved, since there are no contact terms of $O(a)$. These could only enter with the scalar density term, but the result eq. (46) is an algebraic identity, so using it does not introduce additional terms. The improved bilinear $\widehat{S}^{(j k)}$ is, however, only on-shell improved, and this will be crucial in the following.

The result eq. (C3) shows that, if one uses on-shell improved local bilinears and masses in the discretized form of the vector variation of action, then the operators in the associated

18 The form of the $c_{V}$ and $\bar{c}_{V}$ terms for $V_{\mu}^{l a t}(x+\widehat{\mu} / 2)$ is $\partial_{\nu}\left[T_{\mu \nu}(x+\widehat{\mu})+T_{\mu \nu}(x)\right] / 2$, where the derivative is the symmetric difference. 
Ward identities automatically transform with the correct normalization. This is the concrete form of the statement made in the text that the vector Ward identities are automatically satisfied. There are some subtleties, however, in the application of this result, and we spend the remainder of this appendix describing some examples.

\section{Two-point vector Ward identities}

In quenched studies of improvement, the two-point vector Ward identity

$$
\left\langle\left[\left(\widehat{m}_{j}-\widehat{m}_{k}\right) \widehat{S}^{(j k)}(x)-\partial_{\mu} \widehat{V}_{\mu}^{(j k)}(x)\right] J^{(k j)}(0)\right\rangle=O\left(a^{2}\right), \quad j \neq k, \quad x \neq 0
$$

has been used as part of the method employed to determine improvement coefficients [7, 9].

The result eq. (C3) shows, however, that this identity is automatically satisfied, as long as one uses the correctly normalized vector current. Thus it serves only to check the normalization of the vector current, and provides no information on the improvement coefficients of quark masses and scalar bilinears. This point was not appreciated in Ref. 7].

\section{A misleading three-point vector Ward identity}

In Ref. [12], we argued that we could determine $g_{S}$ and $g_{P}$ by enforcing the vector transformation properties of the flavor-singlet scalar and pseudoscalar bilinears, respectively. This is incorrect, as we now show. It is simplest to do this with the pseudoscalar density as it has no mixing with the identity operator.

The identity in question is

$$
\left\langle a^{4} \sum_{\mathcal{V}}\left[\partial_{\mu} \widehat{V}_{\mu}^{(j k)}\right] \widehat{\operatorname{tr} P}(y) J^{(k j)}(z)\right\rangle=O\left(a^{2}\right),
$$

where we work in the chiral limit so that there are no contact terms. We recall that, in the chiral limit, the improved, normalized pseudoscalar density is

$$
\widehat{\operatorname{tr} P}=Z_{P} r_{P}\left[\operatorname{tr} P+a g_{P} \operatorname{Tr}\left(F_{\mu \nu} \widetilde{F}_{\mu \nu}\right)\right]
$$

Our previous argument was that, in order for the LHS of (C5) to be of $O\left(a^{2}\right), O(a)$ contributions from the two terms in $\widehat{\operatorname{tr} P}$ must cancel against each other, thus determining $g_{P}$. In 
fact, it follows from eq. (C3) that both terms separately are automatically of $O\left(a^{2}\right)$, e.g.

$$
\left\langle a^{4} \sum_{\mathcal{V}}\left[\partial_{\mu} \widehat{V}_{\mu}^{(j k)}\right] \operatorname{tr} P(y) J^{(k j)}(z)\right\rangle=O\left(a^{2}\right),
$$

so that the identity (C5) is satisfied for any value of $g_{P}$. In other words, the two terms in $\widehat{\operatorname{tr} P}$ are separately invariant under vector transformations up to $O\left(a^{2}\right)$.

\section{A paradox and its resolution}

Another vector Ward identity used in Ref. [12] was

$$
\begin{array}{r}
\left\langle\left[\left(\widehat{m}_{j}-\widehat{m}_{k}\right) \widehat{S}^{(j k)}(x)-\partial_{\mu} \widehat{V}_{\mu}^{(j k)}(x)\right] \widehat{\mathcal{O}}^{(k j)}(y) J(z)\right\rangle=\langle \\
\left\langle\left[\widehat{\mathcal{O}}^{(k k)}(y)-\widehat{\mathcal{O}}^{(j j)}(y)\right] J(z)\right\rangle \\
+ \text { contact terms }+O\left(a^{2}\right) .
\end{array}
$$

Here we work at non-zero quark mass, so there are contact terms of $O(a)$ because the scalar density is not off-shell improved. These are proportional to $\left(m_{j}-m_{k}\right)$ (without any factors involving $r_{m}$ as in the axial case), because $\widehat{m}_{j}-\widehat{m}_{k} \propto m_{j}-m_{k}+O(a)$. If we use the expressions (16) and (18) for the improved bilinears, and divide through by

$$
Z_{O}\left[1+a \bar{b}_{O} \operatorname{tr} M+a b_{O} m_{k j}\right]
$$

the Ward identity we are enforcing becomes

$$
\begin{aligned}
& \left\langle\left[\left(\widehat{m}_{j}-\widehat{m}_{k}\right) \widehat{S}^{(j k)}(x)-\partial_{\mu} \widehat{V}_{\mu}^{(j k)}(x)\right] \mathcal{O}^{(k j), I}(y) J(z)\right\rangle \\
& =\left\langle\left\{\left[\mathcal{O}^{(k k), I}(y)-\mathcal{O}^{(j j), I}(y)\right]+a b_{O} \frac{\left(m_{k}-m_{j}\right)}{2}\left[\mathcal{O}^{(k k)}(y)+\mathcal{O}^{(j j)}(y)\right]\right.\right. \\
& \left.\left.\quad+a f_{O}\left(m_{k}-m_{j}\right) \operatorname{tr} O(y)\right\} J(z)\right\rangle+ \text { contact terms }+O\left(a^{2}\right) .
\end{aligned}
$$

Previously, we argued that the form of the contact term could be determined by off-shell improving $S^{(j k)}$ with the addition of a term proportional to the equations-of-motion operator (171), as in Refs. [7, 8]. This leads to a contact term proportional to

$$
\text { contact term } \propto\left\langle a\left(m_{k}-m_{j}\right)\left[\mathcal{O}^{(k k)}(y)+\mathcal{O}^{(j j)}(y)\right] J(z)\right\rangle
$$

i.e. of the same form as the $b_{O}$ term. Thus we concluded that $b_{O}$ could not be determined from this Ward identity, but that $f_{O}$ could, since it multiplies an independent operator. 
To see that this is incorrect, we use the general result (C3), from which it follows that

$$
\left\langle\left[\left(\widehat{m}_{j}-\widehat{m}_{k}\right) \widehat{S}^{(j k)}(x)-\partial_{\mu} \widehat{V}_{\mu}^{(j k)}(x)\right] \mathcal{O}^{(k j), I}(y) J(z)\right\rangle=\left\langle\left[\mathcal{O}^{(k k), I}(y)-\mathcal{O}^{(j j), I}(y)\right] J(z)\right\rangle+O\left(a^{2}\right) .
$$

Note that the improvement terms (those proportional to $c_{O}$ ) rotate just as the bare operators, so $\mathcal{O}^{I}$ rotates as a whole. Comparing to eq. (C10) we see that the first term on the RHS is obtained automatically, while the $b_{O}$ and $f_{O}$ terms must either cancel against contact terms or vanish. The discussion above implies that the $b_{O}$ term is canceled, but we must have $f_{O}=0$. This would be a paradoxical conclusion because we used the vector symmetry in the first place to conclude that the $f_{O}$ terms are needed, and yet we have now used the same symmetry to conclude that they vanish.

In fact, this result is wrong. The flaw in the argument is that there is an additional contact term in eq. (C10) proportional to

$$
\left\langle a\left(m_{k}-m_{j}\right) \operatorname{tr} \mathcal{O}(y) J(z)\right\rangle
$$

and this is of the right form to cancel with the $f_{O}$ term. Thus $f_{O}$ does not need to vanish, and indeed cannot be determined from the vector Ward identity, just like $b_{O}$. The operator in the new contact term, $\operatorname{tr} \mathcal{O}$, is allowed because it appears in the operator product of $S^{(j k)}$ and $\mathcal{O}^{(k j)}$, in addition to the other contact term operator $\mathcal{O}^{(k k)}+\mathcal{O}^{(j j)}$. The new operator arises from diagrams in which both the quark and antiquark in the two operators in the product are contracted together - the closed quark loop then coupling to tr $\mathcal{O}$ through intermediate gluons. The presence of this operator shows that the mnemonic of off-shell improvement through the addition of equations-of-motion operators needs to be generalized beyond the considerations of Refs. 7, 8]. A more straightforward approach is simply to enumerate the allowed operators using symmetries. Indeed, one can turn the previous argument around, and use the fact that symmetry implies the presence of the $f_{O}$ terms to imply the existence of the new contact terms.

Finally, we note that these considerations resolve the puzzle concerning the counting of improvement coefficients mentioned in the text. The off-diagonal bilinears require one less improvement coefficient than the diagonal bilinears. How can this be consistent with the fact that a vector transformation rotates the former into the latter (as in the Ward identities just discussed)? The answer is provided by the presence of the new contact term, which 
allows there to be an $f_{O}$ term in the diagonal bilinears but not in the off-diagonal ones.

[1] K. Jansen et al., Phys. Lett. B 372, 275 (1996) arXiv:hep-lat/9512009; M. Luscher, S. Sint, R. Sommer and P. Weisz, Nucl. Phys. B 478, 365 (1996) arXiv:hep-lat/9605038.

[2] K. Jansen and R. Sommer [ALPHA collaboration], Nucl. Phys. B 530, 185 (1998) [Erratumibid. B 643, 517 (2002)] arXiv:hep-lat/9803017.

[3] N. Yamada et al. [JLQCD Collaboration], Phys. Rev. D 71, 054505 (2005) arXiv:hep-lat/0406028.

[4] S. Aoki et al. [CP-PACS Collaboration], arXiv:hep-lat/0508031.

[5] M. Della Morte, R. Hoffmann and R. Sommer, JHEP 0503, 029 (2005) arXiv:hep-lat/0503003.

[6] M. Guagnelli and R. Sommer, Nucl. Phys. Proc. Suppl. 63, $886 \quad$ (1998) arXiv:hep-lat/9709088.

[7] T. Bhattacharya et al., Phys. Lett. B 461, 79 (1999) arXiv:hep-lat/9904011; Phys. Rev. D 63, 074505 (2001) arXiv:hep-lat/0009038.

[8] G. Martinelli et al., Phys. Lett. B 411, 141 (1997) arXiv:hep-lat/9705018.

[9] G. M. de Divitiis and R. Petronzio, Phys. Lett. B 419, 311 (1998) arXiv:hep-lat/9710071.

[10] G. Martinelli, C. Pittori, C. T. Sachrajda, M. Testa and A. Vladikas, Nucl. Phys. B 445, 81 (1995) arXiv:hep-lat/9411010.

[11] G. Martinelli, G. C. Rossi, C. T. Sachrajda, S. R. Sharpe, M. Talevi and M. Testa, Nucl. Phys. B 611, 311 (2001) arXiv:hep-lat/0106003.

[12] T. Bhattacharya, R. Gupta, W. Lee and S. Sharpe, Nucl. Phys. B(Proc. Suppl.)83-84 (2000) 902 arXiv:hep-lat/9909092.

[13] T. Bhattacharya, R. Gupta, W. j. Lee, S. R. Sharpe and J. M. S. Wu, Nucl. Phys. Proc. Suppl. 129 (2004) 441 arXiv:hep-lat/0309087.

[14] M. Della Morte, R. Hoffmann, F. Knechtli, R. Sommer and U. Wolff, JHEP 0507, 007 (2005) arXiv:hep-lat/0505026.

[15] S. Sint and R. Sommer, Nucl. Phys. B 465, 71 (1996) arXiv:hep-lat/9508012.

[16] R. F. Dashen, Phys. Rev. D 3, 1879 (1971);

M. Creutz, Phys. Rev. Lett. 92, 201601 (2004) arXiv:hep-lat/0312018. 
[17] S. Sint and P. Weisz, Nucl. Phys. B 502, 251 (1997) arXiv:hep-lat/9704001; Nucl. Phys. Proc. Suppl. 63, 856 (1998) arXiv:hep-lat/9709096.

[18] M. Testa, JHEP 9804, 002 (1998) arXiv:hep-th/9803147.

[19] P. E. L. Rakow, Nucl. Phys. Proc. Suppl. 140, 34 (2005) arXiv:hep-lat/0411036.

[20] T. Bakeyev, M. Gockeler, R. Horsley, D. Pleiter, P. E. L. Rakow, G. Schierholz and H. Stuben [QCDSF-UKQCD Collaboration], Phys. Lett. B 580, 197 (2004) arXiv:hep-lat/0305014.

[21] L. Maiani, G. Martinelli, M. L. Paciello and B. Taglienti, Nucl. Phys. B 293, 420 (1987); for further discussion see R. Gupta, C. F. Baillie, R. G. Brickner, G. W. Kilcup, A. Patel and S. R. Sharpe, Phys. Rev. D 44, 3272 (1991).

[22] M. Bochicchio, L. Maiani, G. Martinelli, G. C. Rossi and M. Testa, Nucl. Phys. B 262, 331 (1985).

[23] D. Pekurovsky and G. Kilcup, Phys. Rev. D 64, 074502 (2001) arXiv:hep-lat/9812019.

[24] J. I. Noaki et al. [CP-PACS Collaboration], Phys. Rev. D 68, 014501 (2003) arXiv:hep-lat/0108013.

[25] T. Blum et al. [RBC Collaboration], Phys. Rev. D 68, 114506 (2003) arXiv:hep-lat/0110075.

[26] R. Frezzotti and G. C. Rossi, JHEP 0408, 007 (2004) arXiv:hep-lat/0306014.

[27] R. Frezzotti, P. A. Grassi, S. Sint and P. Weisz, Nucl. Phys. Proc. Suppl. 83, 941 (2000) arXiv:hep-lat/9909003;

R. Frezzotti, P. A. Grassi, S. Sint and P. Weisz [Alpha collaboration], JHEP 0108, 058 (2001) arXiv:hep-lat/0101001.

[28] F. Farchioni et al., PoS LAT2005, 072 (2005) arXiv:hep-lat/0509131. 